\title{
Integrated analysis of genetic, behavioral, and biochemical data implicates neural stem cell-induced changes in immunity, neurotransmission and mitochondrial function in Dementia with Lewy Body mice
}

\author{
Anita Lakatos ${ }^{1,2+}$, Natalie R. S. Goldberg ${ }^{1,2+}$ and Mathew Blurton-Jones ${ }^{1,2,3^{*}}$ (D)
}

\begin{abstract}
We previously demonstrated that transplantation of murine neural stem cells (NSCs) can improve motor and cognitive function in a transgenic model of Dementia with Lewy Bodies (DLB). These benefits occurred without changes in human a-synuclein pathology and were mediated in part by stem cell-induced elevation of brain-derived neurotrophic factor (BDNF). However, instrastriatal NSC transplantation likely alters the brain microenvironment via multiple mechanisms that may synergize to promote cognitive and motor recovery. The underlying neurobiology that mediates such restoration no doubt involves numerous genes acting in concert to modulate signaling within and between host brain cells and transplanted NSCs. In order to identify functionally connected gene networks and additional mechanisms that may contribute to stem cell-induced benefits, we performed weighted gene co-expression network analysis (WGCNA) on striatal tissue isolated from NSC- and vehicle-injected wild-type and DLB mice. Combining continuous behavioral and biochemical data with genome wide expression via network analysis proved to be a powerful approach; revealing significant alterations in immune response, neurotransmission, and mitochondria function. Taken together, these data shed further light on the gene network and biological processes that underlie the therapeutic effects of NSC transplantation on a-synuclein induced cognitive and motor impairments, thereby highlighting additional therapeutic targets for synucleinopathies.
\end{abstract}

Keywords: Synuclein, Neural stem cells, Transplantation, Dopamine, Lysosome, Autophagy, Genomics, Glutamate, Memory

\section{Introduction}

For the great majority of neurodegenerative diseases, neuronal damage and loss occur long before clinical symptoms first become apparent [79, 102]. Likely for this reason therapies designed to target upstream pathological initiators of disease have thus far failed in clinical trials of symptomatic patients $[30,90]$ In contrast, the emerging field of stem cell transplantation may offer a

\footnotetext{
* Correspondence: mblurton@uci.edu

${ }^{\dagger}$ Equal contributors

${ }^{1}$ Sue and Bill Gross Stem Cell Research Center, University of California, Irvine, USA

${ }^{2}$ Institute for Memory Impairments and Neurological Disorders, University of California, Irvine, USA

Full list of author information is available at the end of the article
}

potentially powerful approach to restore some aspects of brain function that have previously been lost [66]. Neural stem cells (NSCs) in particular have the potential to develop into the three principle cell-types of the brain; neurons, astrocytes, and oligodendrocytes, and can also renew themselves through asymmetrical cell division [92]. Although great progress has been made toward our understanding of NSCs and their potential application in Central Nervous System (CNS) disorders, much still needs to be determined regarding the biology of these cells and their complex interactions with endogenous host tissue and disease pathology in order to maximize therapeutic benefits $[2,15,23,24,40,41,63,96]$. 
We previously examined the effects of haplotypematched murine NSC transplantation in a transgenic model of Dementia with Lewy Bodies (DLB) that overexpresses wild-type human $\alpha$-synuclein (ASO mice) [41]. Interestingly, we found that NSCs could significantly improve both motor and cognitive function 1 month after transplantation into the striata of aged ASO mice. These benefits, however, were not accompanied by any changes in Lewy body-like $\alpha$-synuclein inclusions. Instead, behavioral recovery was associated with significant increases in brain-derived neurotrophic factor (BDNF), tyrosine hydroxylase activity, and glutamate type I transporter (GLT-1). Furthermore, reduction of BDNF within NSCs prevented the cognitive and motor benefits of transplantation, suggesting that neurotrophic effects of NSCs played a principal role in recovery. However, in a complementary approach, we found that viral delivery of BDNF alone only partially mimicked the effects of NSC transplantation; improving motor function but failing to significantly improve cognition [41]. Thus, we concluded that NSCs likely influence a broader set of mechanisms to affect host neuronal function and behavior. In order to identify these other potential regulatory networks involved in NSC-induced functional recovery, we have now examined whole genome gene expression in striatal samples isolated from these same mice [41].

Network analysis, a quasi-dynamic modelling of transcriptomics, offers a powerful approach to gain insight into the biological mechanisms of disease and treatment related recovery $[3,58,70,73,74,103,112]$. Combining this genomic network approach with quantitative phenotype-based analysis can in turn help to unravel the complexity of neurodegenerative diseases with considerable statistical power $[45,83]$. Therefore, we implemented a systems biology approach [80] that combines quantitative phenotypes with genome wide gene expression in a network analysis to gain further insight into the mechanisms that underlie stem cell-mediated functional recovery. First, we constructed potential regulatory networks using weighted gene co-expression network analysis (WCGNA), and subsequently integrated these networks with continuous disease-related quantitative behavioral and biomarker measurements. Using this approach, we successfully identified several candidate gene networks and corresponding biological mechanisms relevant to DLB disease states and NSC engraftment. Our findings indicate that NSC transplantation robustly modifies multisystem neurotransmission, mitochondrial and lysosomal function, and immune responses in close association with improved cognitive and motor function. These results therefore greatly enhance our understanding of the mechanisms by which neural stem cell transplantation modulates neuronal and behavioral function, and point toward new disease- and recovery-associated networks that warrant further consideration.

\section{Materials and methods}

Animals, stem cell transplantation, and behavioral tasks

All aspects of the animal genetic background, stem cell engraftment, behavioral tasks and the biomarker biochemical assays are detailed in Goldberg et al. [41]. Briefly, all animal experiments were performed in strict accordance with the University of California, Irvine animal use regulations and the NIH guide for the Care and Use of Laboratory Animals. Age- and sex-matched transgenic mice over-expressing human wild-type $\alpha$ synuclein (PDGF- $\beta$-ASO line D, ASO) and wild-type littermates maintained on a congenic C57B6/J background were examined. Hippocampal/cortical GFP-expressing mouse neural stem cells (NSCs) were microdissected from syngeneic GFP-transgenic mice at postnatal day 1, grown as adherent monolayers, and transplanted at passage 15 as previously described $[15,75]$. NSCs remained on ice for the duration of the transplantation procedure and retained $89-94 \%$ viability as assessed by trypan-blue exclusion of remaining cells following transplantation [41]. Wild-type (WT) and transgenic littermates (ASO) were randomly divided into groups and received intrastriatal injections of either saline (Veh) or 100,000 haplotype-matched NSCs and WT and ASO transplants were alternated to avoid any potential confounding effect of transplantation time. The combination of genotype and treatment resulted in four experimental groups: WT-Veh, WT-NSC, ASO-Veh, ASO-NSC. All behavioral tasks were performed and analyzed by a researcher blinded to genotype and treatment began 30 days after NSC transplantation; and followed standard protocols to perform Novel Object Recognition (NOR), Novel Place Recognition (NPR), Rotarod, and Beam Transversal tasks [5, 6, 11, 71]. Following behavioral assessment, mice were sacrificed by transcardial perfusion with $0.01 \mathrm{M}$ phosphate buffered saline and then brains were hemisected to provide tissue for histological, biochemical, and RNA analyses.

\section{RNA extraction and biochemical analyses}

For biochemical and gene expression analyses, brains were flash frozen immediately following dissection and maintained in a semi-frozen state using dry ice chips during microdissection of the dorsal striatum and all dissections were performed within $1 \mathrm{~min} /$ brain. Tissue was then processed to isolate both mRNA and protein via TRIzol $^{\odot}$ using the manufacture's guidelines (Life Technologies, Inc., Carlsbad CA). RNA quality control analyses were performed by the University of California, Irvine Genomics High-Throughput Facility at the Chao Family Comprehensive Cancer Center and included 
assessment of RNA concentration, 260/280 and 260/230 ratios and calculation of RNA integrity number (RIN) (Additional file 1). Five mice per group with the highest quality RNA (RIN >9) were analyzed for the current gene expression study. Protein concentrations were determined by Bradford assay and normalized samples compared by SDS-PAGE Western blot. Antibodies were used to detect mature and proBDNF (Santa Cruz Biotech \#sc-546), phosphorylated Tyroxine Hydroxylase (pSer31TH, Cell Signaling, \#3370), and Glial High Affinity Glutamate Transporter (GLT-1, Slc1a2, Abcam, \#ab106289) as detailed in Goldberg et al. [41]. Relative signal intensity of grayscale images was then quantified by ImageJ software and once all values were obtained sample identification was decoded. The behavioral and biomarkers measurements described above and detailed in [41] were then used as quantitative phenotypes in the WGCNA. Additional file 2: Figure S1 summarizes the experimental design.

\section{Affymetrix gene array processing}

All animals were sacrificed and total RNA extracted from microdissected striatum as described above. Sample purity and concentration were verified by Bioanalyzer (Agilent). All 20 RNA samples were processed on a GeneChip ${ }^{\circ}$ Mouse Gene 2.0 ST Array (Affymetrix, Santa Clara, CA) by the UCI Genomics High-Throughput Facility following the manufacture's guidelines. All CEL files were subjected to background correction, normalization and 'core' summarization using the robust multiarray analysis (RMA) algorithm implemented in Bioconductor package "oligo 1.34.2". All probes were mapped to genes based on Bioconductor package "mogene20sttranscriptcluster.db 8.4.0". After initial quality control (QC) analysis including RNA degradation assessment (Additional file 2: Figure S2) and clustering (Additional file 2: Figure S3), one sample was marked as an outlier and omitted from subsequent analyses. Then, array probes were filtered" for unique Entrez IDs and the most variable genes across samples by applying the interquartile range (IQR) variance filter implemented in Bioconductor package "genefilter 1.52.1. Subsequently, $50 \%$ of genes were filtered out from the original dataset leaving approximately 12,300 most variable genes for downstream analysis (detailed parameters can be found in Additional file 3). To control for potential confounding effects, all samples were adjusted for sex and litter effect by using the SampleNetwork1.07 tool [77] prior to gene network construction (Additional file 2: Figure S3.C and D).

\section{Weighted gene correlation network analysis (WGCNA)} WGCNA (package version 1.51) implemented in $\mathrm{R}$ tool (version 3.2.3) was performed on all samples that passed
QC using standard methods [58]. The function "blockwiseModules" was used as described in [76] to assign each gene to a "signed" network (module) with the following parameters; softPower "20", corType "bicor", deepSplit "4", minModuleSize " 50 ", minKMEtoStay "0", mergeCutHeight "0.25”, "detectCutHeight "0.99995" (code for module construction can be found in Additional file 3). Then, gene expression was summarized into module eigengene (ME) as the first principal component $(\mathrm{PC})$ of the entire module gene expression. Consequently, the module specific PCs were correlated by using the "bi-weight mid-correlation" (bicor) method with continuous measurements of behavioral phenotypes and biomarkers. A correlation was considered significant at $p<0.05$ with absolute correlation $>0.5$. The result of analysis was visualized by "ggplot2" [49], "ggtree" [109] and "circos plot" [55]. For each gene, intramodular connectivity (module membership) often referred to as "node degree" was computed to determine genes with the most numerous connections called "hub" genes for each module [58, 59]. Gephi [8], Igraph [28], RedeR [22] tools were used to depict the relationship between hub genes in some of the modules. Potential important genes were selected by plotting the absolute values of the computed intramodular connectivity and the gene significance determined by the strength of correlation to the phenotype of interest. Genes that scored high on both scales were designated to be candidate genes for stem cell treatment related biological mechanisms.

\section{Gene Set enrichment analysis (GSE)}

To determine whether a set of genes was differentially expressed between two conditions based on a nonparametric (Kolmogorov-Smirnov) statistical modeling, we utilized Gene Set Enrichment Analysis (GSE) [94]. We used this approach to assess whether a module as a whole was significantly down- or up-regulated in one experimental condition versus the other. Thus, each module was transformed into a geneset and tested with the stand-alone version of the GSEA tool [93]. At first, all modules were examined by contrasting ASO-NSC versus ASO-Veh conditions. Subsequent experiments tested all modules in additional 4 comparisons: ASONSC versus WT-Veh, WT-NSC versus WT-Veh, ASOVeh versus WT-Veh and ASO-NSC versus WT-NSC. The results were considered significant at an FDR qvalue $<0.05$. (The result is summarized in Additional file 4 and GSEA parameters are included in Additional file 3). The altered modules and pathways in comparison between ASO-NSC versus WT-Veh, ASO-NSC versus WT-NSC and ASO-Veh versus ASO-NSC are summarized in Additional file 5. 


\section{In silico functional annotation}

Biological relevance of each module was tested by performing serial gene enrichment analyses. All tools were based on either hypergeometric test, Fisher's exact test or a combined score test. At first, we identified modules with cell type specific expression patterns by using the Specific Expression Analysis (SEA) online tool [108]. To determine whether modules corresponded to particular subcellular components, we mined the subcellular organelle database OrganelleDB [105]. We also assed the exosomal content of each module with the FunRich tool [81], exploiting the Extracellular Vesicles database [52]. Next, we performed gene ontology and pathway analysis using a web based tool, Enrichr [56], as well as ClueGo and CluePedia [14] implemented in Cytoscape and supplemented with enrichment analysis in WGCNA. Complementary to these analyses, our functional interpretation of gene modules exploited several biological databases, including the Barres RNAseq database [110] and Innate Database [18]. Additional file 2: Figure S1B outlines the network analysis and annotation workflow.

\section{Results}

We previously demonstrated that transplantation of murine NSCs leads to significant improvements in both motor and cognitive function in a transgenic model of DLB [41]. In addition, we found that these improvements correlated with altered dopaminergic and glutamatergic signaling and were driven in part by increases in mature BDNF protein. In the current study, we aimed to build upon these findings to identify and better understand the molecular and transcriptional changes that underlie these improvements. We therefore applied a co-expression network analysis in which we combined quantitative measurements of behavioral tasks and biomarker proteins with genome wide gene expression. This approach served two main purposes: 1) to lend considerable statistical power to interpreting associations between genomic and phenotypic quantitative measurements; and 2) identify tightly correlated gene networks that may reveal cell and tissue specific biological mechanisms through co-expression analysis.

WGCNA analysis reveals 11 gene modules associated with phenotypic traits

We collected RNA from mouse striatum from 4 treatment groups $(n=5)$ and obtained high-throughput gene expression profiles on an Affymetrix microarray. Following microarray processing, 19 of 20 transcriptomes passed quality control and were subjected to network analysis implemented in WGCNA [58]. In an unsupervised manner, all transcriptomes were initially combined and partitioned into correlated gene sets called transcription modules. Approximately 12,000 variable genes with Entrez IDs yielded 24 sets of tightly correlated modules (Additional file 2: Figure S4). The overall gene expression of each module was then collapsed into the first principal component of gene expression variance called the "module eigengene" (ME). In a subsequent analysis, MEs were used to measure the strength of correlation between 24 modules and quantitative phenotypes (Additional file 2: Figure S5). Thirty one correlations from a total of 192 passed the statistical threshold of $p<0.05$ and exhibited correlations less than $r<-0.5$ or larger than $r>0.5$ with one or more behavioral tasks or biomarkers, yielding 13 significant modules of the initial 24 (Fig. 1a, Additional file 6). Since the network construction and correlational analysis were conducted in a blinded manner, we first performed gene set enrichment analysis (GSEA) to specifically address whether the modules were differentially expressed in ASO mice in response to Vehicle or NSC treatment. We found 11 of the 13 modules were significant and differentially expressed between ASO-NSC and ASO-Veh animals (Fig. 1b, Additional file 4). Table 1 summarizes the main characteristics of the significant modules. The most significant relationship $(p<10 \mathrm{E}-04$, correlation $=0.78)$ was found between Module 2 and the biomarker pSer31TH which measures the phosphorylation of tyrosine hydroxylase $(\mathrm{TH})$ at Serine 31, a strong indication of enzyme activity and dopamine synthesis [86]. Module 1, Module 2 , and Module 17 had the largest number of significant correlations with behavioral or biomarker phenotypes (7) while biomarker pSer31TH was correlated with the highest number of modules (7) followed by performance in the Novel Object recognition task (6) (Fig. 1b, Additional file 2: Figure S5). Interestingly, most of the 11 modules especially the ones related to stem cell behavior were found similarly regulated in WT-NSC and ASO-NSC (M1, M2, M11, M13, M15, M16, M17, M18; Additonal files 7, 8, 9, 10, 11, 12, 13, 14, 15, 16, 17, 18, $19,20)$ suggesting common properties of the stem cells on cellular function irrespective of disease background (Additional files 4 and 5). To determine potential mechanisms by which functional recovery occurred following NSC transplantation, we next investigated the biological significance of each module by performing in silico functional annotation.

\section{NSCs influence dopaminergic, GABAergic, and glial cell types within the striatum}

Module 1 (M1, turquoise) contained the greatest number of genes (1848) and showed significant positive ME correlation with Novel Object recognition $(p<5.6 \mathrm{E}-04)$, Novel Place recognition $(p<1.1 \mathrm{E}-02)$, and the biomarkers pSer31TH $(p<1.4 \mathrm{E}-04)$ and mature BDNF (mBDNF; $p<2.0 \mathrm{E}-03$ ) (Fig. 2a, Additional file 2: Figure S4). The large number of genes in this module likely 


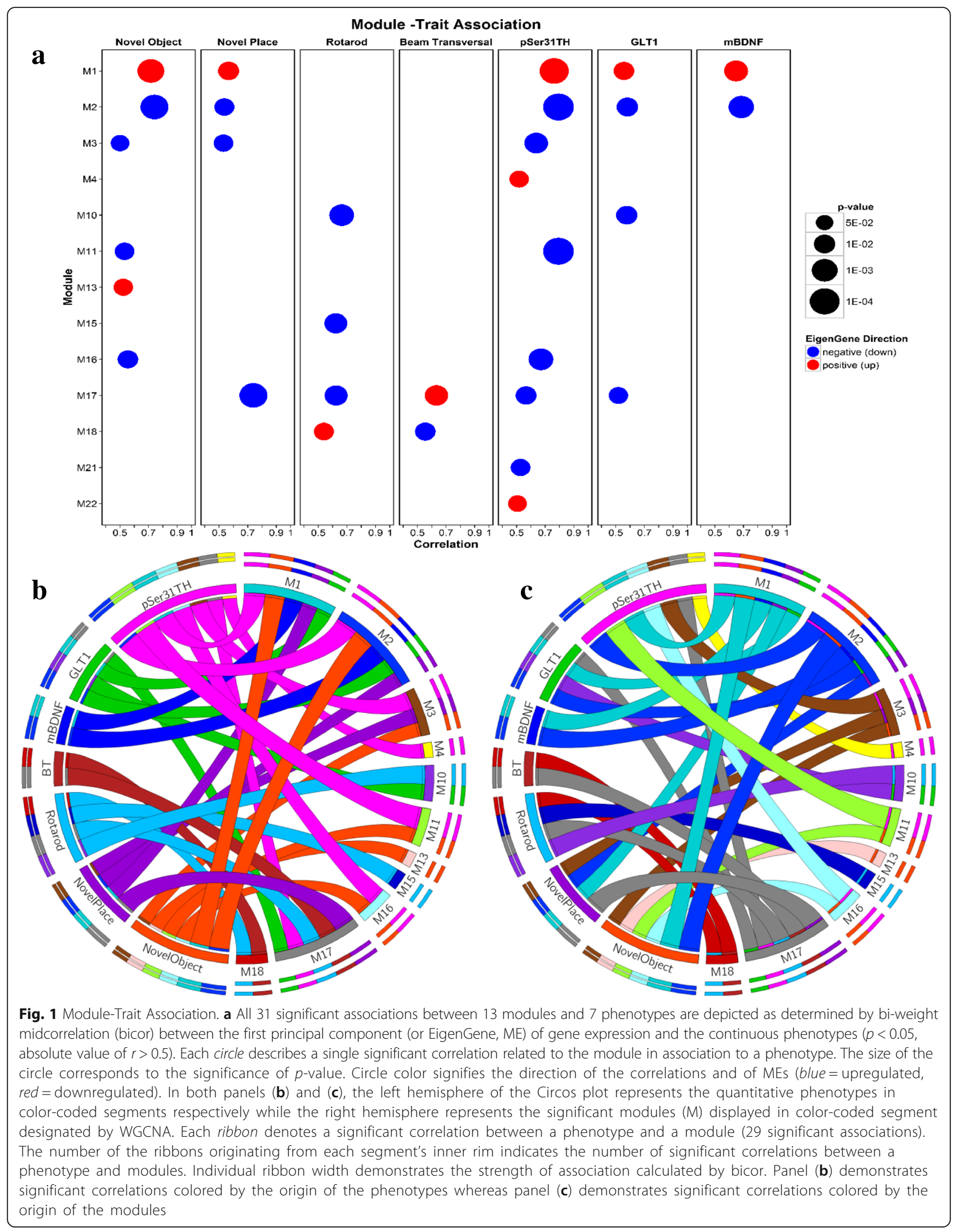




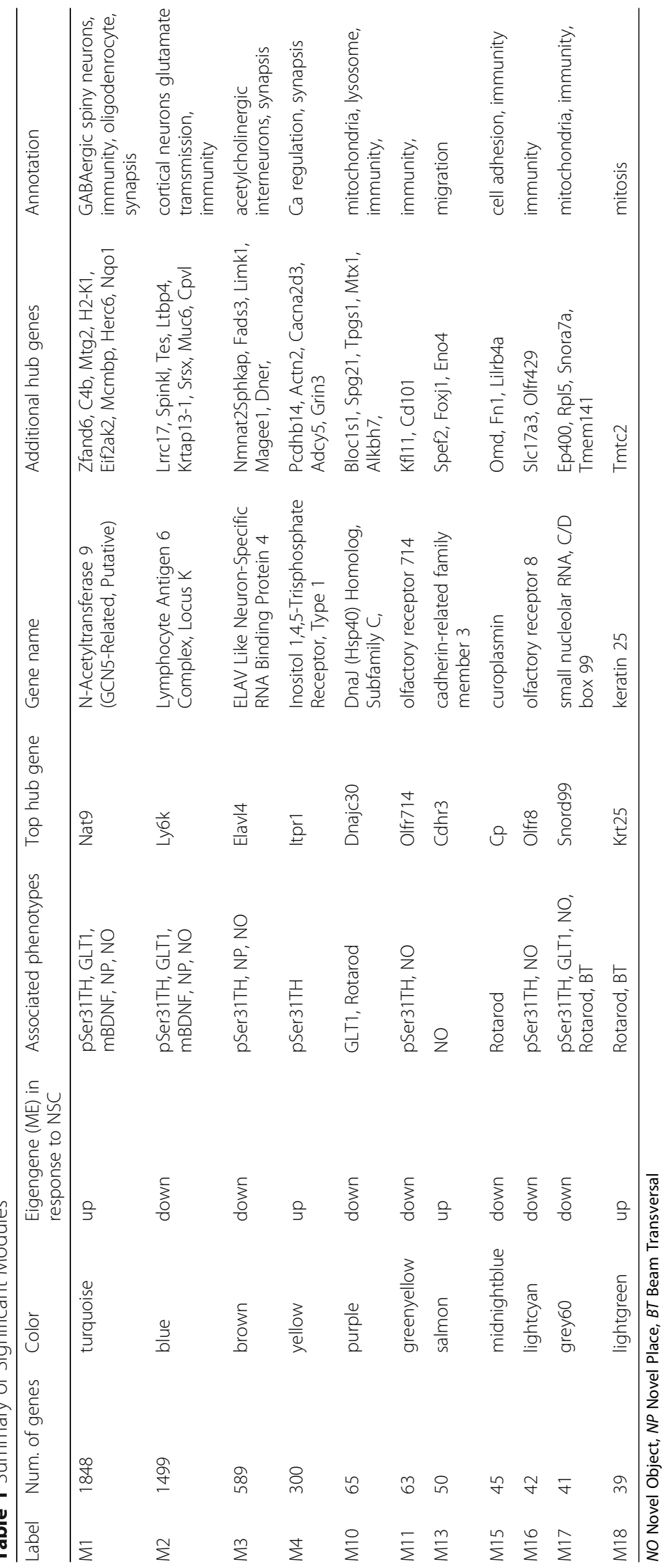




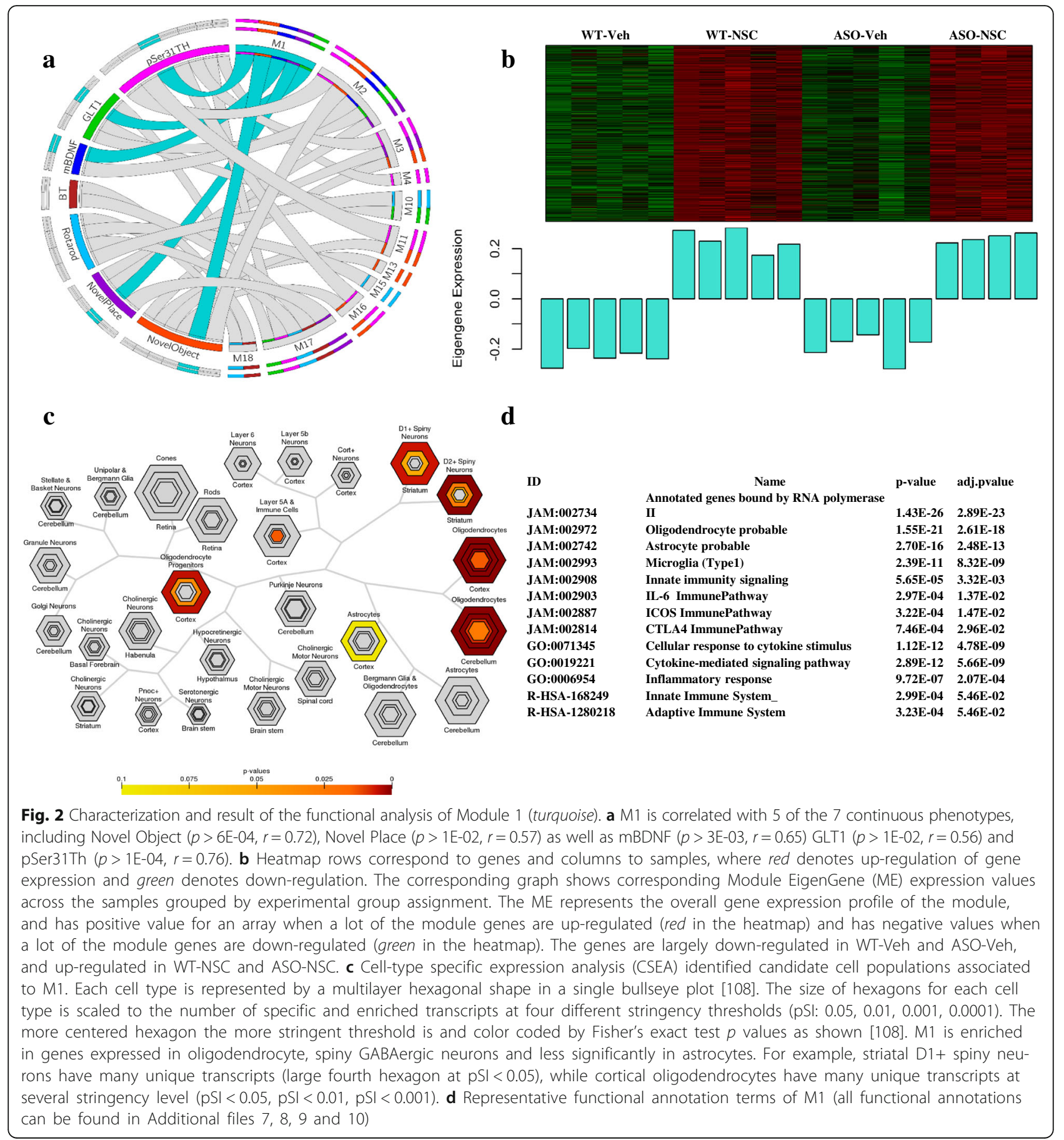

reflects the complexity of network changes that results from incorporating one biological system (NSCs) into another multicellular biological system (mouse CNS). Although large modules such as M1 can make it difficult to understand the precise relationship between hub and connected genes, this module appears to primarily represent NSC-induced changes in gene expression that are largely independent of $\alpha$-synuclein genotype. The top hub gene in M1, Nat9 (N-Acetyltransferase 9) has previously been associated with autoimmune functions in the peripheral system, but is also highly expressed in microglia and implicated in CNS development [29, 44, 114]. Overall this module exhibited a significant upregulation of gene expression in response to NSC treatment in both WT and ASO mice (Fig. 2b). Interestingly, M1 also included significant gene sets related to stem cell biology, identifying several biological processes that are preferentially activated during stem cell proliferation and 
glial cell differentiation (Additional files 7, 8, 9 and 10). It follows that this module appears to be strongly related to NSC-related cellular changes, and is less influenced by $\alpha$-synuclein genotype (Fig. $2 b$ ). The cell type specific enrichment analysis of Module 1 showed a strong association with D1 and D2 GABAergic spiny neurons (Fig. 2cd), and tissue specificity pointed toward substantia nigra and striatal regions, suggesting an influence of NSCs on the nigrostriatal system, a key region of degeneration in Parkinson's disease and DLB. Cell type specific analysis also highlighted oligodendrocytes and astrocytes, which agrees with our prior histological findings that the majority of the engrafted NSCs differentiated into glia with approximately $26 \%$ cells expressing the astrocyte specific marker (GFAP), another $\sim 32 \%$ expressing the oligodendroglial marker OLIG2, but only $\sim 7 \%$ expressing the early neuronal marker doublecortin (DCX) [41].

\section{Influence of NSCs on immune response}

In addition to revealing cell types and neurocircuitry of interest, module M1 also revealed an association with both innate and adaptive immunity. M1 was significantly enriched in pathways and gene ontology terms (GO) referring to inflammation and immunity (R-HSA168249; R-HSA-1280218; R-HSA-1280215, JAM:003031, GO:0009615, GO:0001817, GO:0045088, GO:0060337) (Fig. 2d) as well as microglia activation (JAM:002993). Gene sets related to regulatory $\mathrm{T}$ lymphocytes (Treg) and cytokine suppression signaling (JAM:002887, JAM:002814, JAM:002974) as well as the transcription factor FOXO1, a master regulator of Treg cells [78], were also significantly enriched (Additional files 7, 8, 9 and 10). Besides significant Foxo1 regulated genes and the CTLA immune pathway (JAM:002814), molecular markers associated with Treg functional activity such as IL10ra [62] and Cd25 (Il2ra) [85] were also components of this module. These findings are intriguing in light of a recent report that NSC-induced activation of Tregs may play a critical role in recovery and remyelination in a model of Multiple Sclerosis [23] and growing evidence that CNS cell transplantation can modulate both innate and adaptive immunity and vice versa $[39,61,82]$. These data therefore suggest that NSC-induced processes including proliferation, glia differentiation and immune modulation, may help to create a neuroprotective environment that increases dopamine transmission and behavioral performance.

The role of exosomes in cell communication and intercellular transfer of bioactive molecules has been recognized in tissues outside the nervous system and is best established among cells of the immune system, where exosomes have been demonstrated to modulate antigen presentation and the immune response [31]. Secretion of exosomes containing regulatory factors by both NSCs and newly formed neuroglia could therefore alter microenvironment cues to modulate both immune and neuronal function $[9,27,54]$. We therefore compared the content of the modules to Vesiclepedia, a compendium of molecular data of extracellular vesicles including ectosomes, exosomes and apoptotic bodies [52]. Interestingly, M1 was associated with exosomal RNAs and proteins, as was also significantly associated with the GO term 'external side of plasma membrane' (GO:0009897). Taken together, M1 functional annotation revealed biological processes related to GABAergic spiny neurons, nigrostriatal dopaminergic systems, stem cell glial differentiation, immunity and exosomes.

In addition to M1, modules M11 and M16 further implicate attenuated immunity. Module 11 (M11, greenyellow) included 63 genes and demonstrated a significant negative correlation with Novel Object task $(p<$ 1.9E-02) and pSer31TH $(p<9.7 \mathrm{E}-03)$ (Fig. 1 , Additional file 2: Figure S5) and was significantly downregulated in response to NSCs treatment in both WT and ASO mice (Additional files 4 and 5). This module is diverse but is enriched in terms related to G-protein coupled receptor signaling (GO:0007186), oxidoreductase activity (GO:0016717), and immune and inflammatory response (GO:0006954, GO:0006955) (Additional files 7, 8, 9 and 15). Likewise, module 16 (M16, ligthcyan) consisting of 42 genes demonstrated significant negative correlation with the Novel Object task $(p<1.3 \mathrm{E}-02)$ and the pSer31TH biomarker $(p<1.6 \mathrm{E}-03)$ (Fig. 1, Additional file 2: Figure S5). ME was significantly upregulated in ASO mice but was in turn downregulated by NSCs treatment. This module is associated with a number of immunological processes including cytokine-cytokine receptor interactions, and interleukin signaling pathways (Additional files 7, 8, 9 and 18). To further confirm the role of innate immunity in stem cell-mediated gene expression changes, we examined patterns of innate immunity related gene expression (InnateDB) [18] in our dataset. Clustering revealed a sharp separation between WT and ASO mice and an overall grouping of NSC transplanted mice (Additional file 2: Figure S6). Thus, it appears that NSC transplantation dramatically alters immune function in both WT and ASO brains in a manner that correlates well with cognitive function and dopaminergic signaling.

\section{Both a-synuclein and NSC transplantation influence cell migration}

Another module that exhibited a significant positive correlation with the Novel Object cognitive task was Module 13 (M13, salmon; $p<2.1 \mathrm{E}-05$ ) (Fig. 1, Additional file 2: Figure S5) which included 50 genes and primarily implicates cell migration. This module was downregulated in ASO mice compared to WT, and upregulated in 
response to NSC transplantation (Additional files 4 and 5) in both WT and ASO mice and was associated with neuronal migration (hs_Lis1Pathway), axoneme assembly (GO:0035082), cytoplasmic dynein complex (GO:0005868, GO:0044782, GO:0044782) movement of cell or subcellular components (GO:0006928) and NSC migration inducing cytokines (e.g., CXCR4) (Additional files 7, 8, 9 and 16). The hub gene in M13 is Cdhr3 (Cadherin-Related Family Member 3) which is involved in cell-cell adhesion (GO:0098609, GO:0007156) including epithelial polarity, cell-cell interactions and differentiation [48]. In addition, Cdhr3 has been implicated in tissue morphogenesis, coordinated cell movements, and the induction and maintenance of structural and functional cell and tissue polarity [43]. This module therefore likely reflects the motility of the transplanted NSCs or host immune cells throughout the striatum and into the adjacent cortices in accordance with our previously published findings [41]. Since Module 13 expression was generally downregulated in ASO compared to WT mice, it is possible that $\alpha$-synuclein pathology also inhibits cell migration.

\section{Altered neurotransmitter and calcium signaling}

Module 4. (M4, yellow) represents an especially interesting group of genes as this module was significantly downregulated in ASO mice compared to WT and NSC treatment reversed the direction of gene expression (Additional file 1) suggesting that NSC transplantation partially rescue an $\alpha$-synuclein-driven perturbation of these genes. M4 consists of 300 genes and revealed a significant positive correlation with the biomarker pSer31TH $(p<2.3 \mathrm{E}-02)$ (Fig. 1, Additional file 2: Figure S5). The top hub gene in this module Itpr1, Inositol 1,4,5-Trisphosphate Receptor Type 1 , is a member of the main endoplasmic reticulum (ER) $\mathrm{Ca} 2+$ - release channel family that modulates intracellular calcium signaling [12]. Interestingly, mutations in Itpr1 have been associated with another movement disorder; spinocerebellar ataxia type 15 [47]. The module significant functional annotation is related to calcium signaling (hsa04020), dopaminergic synapses (hsa04728), synaptic transmission (GO:0007268), postsynaptic density (GO:0045211, GO:0045211), locomotor behavior (GO:0007626), ion channel complex (GO:0034702) and voltage-gated cation channel activity (GO:0022843) (Additional files 7, 8, 9 and 13). The correlation of Module 4 with the pSerTH31 biomarker indicates a particular influence of both $\alpha$-synuclein and NSC transplantation on striatal dopaminergic synapses. In accordance with these findings it has previously been reported that the synaptic accumulation of $\alpha$-synuclein triggers the redistribution of several presynaptic proteins including SNAP-25, syntaxin-1, and synaptobrevin- 2 resulting in an age-dependent reduction in dopamine release [20,37]. It is also noteworthy that, one of the signaling events that can influence the ERK cascade which phosphorylates Ser31 of TH, is a change in free intracellular calcium concentrations $[1$, 26]. This module may therefore represent Calcium mediated signaling that leads to improved synaptic plasticity and phosphorylation of $\mathrm{TH}$ by MAPK cascades in postsynaptic terminal of dopaminergic projection neurons. Module 2 (M2, blue) also appeared to be indicative of changes in synaptic transmission and included 1499 genes that showed significant negative correlations with the Novel Object $(p<2.8 \mathrm{E}-04)$ and Novel Place tasks $(p<1.7 \mathrm{E}-02)$, as well as the biomarkers pSer31TH $(p<5.2 \mathrm{E}-05), \operatorname{mBDNF}(p<1.2 \mathrm{E}-03)$, and GLT1 $(p<9.0 \mathrm{E}-03)$ (Fig. 3a, Additional file 2: Figure S5). This module was also significantly downregulated in response to NSC transplantation in both WT and ASO mice (Fig. 3b). Interestingly, M2 is highly specific for cortical neurons (Fig. 3c) and enriched in genes related to glutamatergic synaptic transmission (Fig. 3d; GO:1900449, GO:0032281, GO:0007409), neuronal projection (GO:0043005, GO:0048812) and immune cells (GO:0002828) (Additional files 7, 8, 9 and 11). This module is also significantly correlated to the GLT-1 biomarker $(p<3.0 \mathrm{E}-03)$ (Fig. 3a), and is therefore likely related to our prior finding that NSC transplantation modulates the expression of both neuronal and astrocytic glutamate transporters; VGLUT1 and GLT-1 [41].

Clustering closely with M2 (Additional file 2: Figure S8), Module 3 (M3, brown) included 589 genes, and showed significant negative correlations with Novel Object recognition $(p<2.8 \mathrm{E}-02)$, Novel Place recognition $(p<1.9 \mathrm{E}-02)$, and pSer31TH $(p<3.0 \mathrm{E}-03) \quad$ (Fig. 4a, Additional file 2: Figure S5). This module also showed a trend toward upregulation in ASO mice but was significantly downregulated in response to NSCs treatment in both WT and ASO mice (Fig. 4b). Interestingly, M3 was enriched in neuron specific genes (JAM:002958) indicative of striatal cholinergic interneurons and cell-type specific expression analysis (CSEA) revealed enrichment of genes expressed in cholinergic neurons (Fig. 4c). Significant GO terms for this module were related to neuronal (GO:0022008, GO:0030182, GO:0048699) and synaptic function (GO:0007268, GO:1902630) (Additional files 7, 8, 9 and 12). As cholinergic interneurons play an important role in regulating striatal output, these data could suggest that NSC-induced changes in cholinergic function may play an important role in motor or cognitive recovery. Interestingly, the top hub gene for this module is Elavl4 (HuD/ELAV like protein 4) (Fig. 4d, Additional file 2: Figure S7). Elavl4 is 


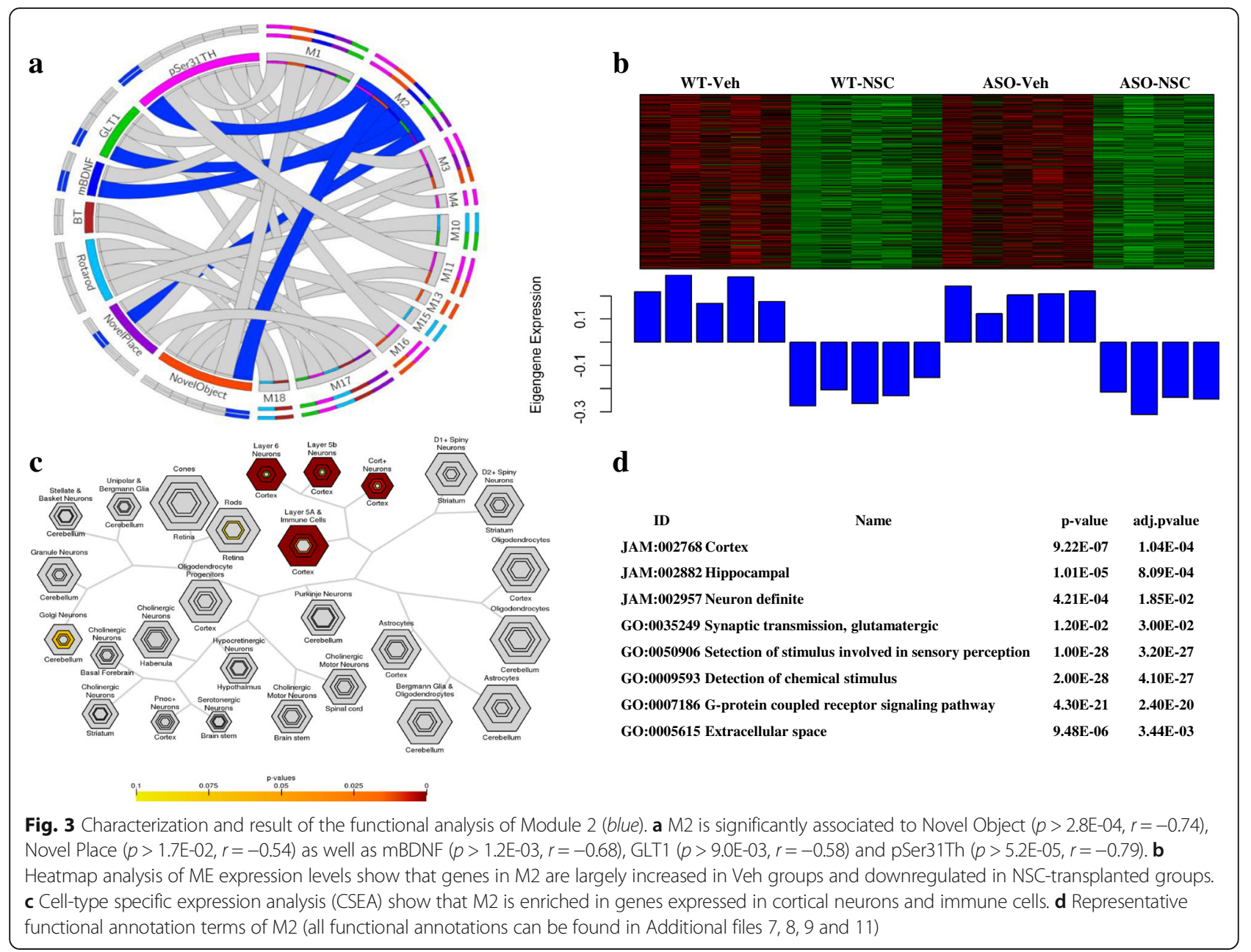

implicated in neuron-specific RNA processing, dendritic morphogenesis, and learning and memory [19]. Furthermore, genetic variants in human ELAVL4 gene have been associated with age of onset in Parkinson's disease (PD) [35].

\section{a-synuclein pathology and NSC transplantation impact} mitochondrial and lysosomal-related gene expression Mitochondrial dysfunction and impairments in lysosomal function and mitophagy have been strongly implicated in the pathogenesis of Parkinson's disease and other synucleinopathies $[13,70,97,107,111]$. It is therefore extremely interesting that Module 10 (M10, purple), includes 65 genes that together implicate mitochondrial and lysosomal function, oxidative stress, and apoptosis and this module exhibits a significant negative correlation with Rotarod $(p<1.9 \mathrm{E}-03)$ and the biomarker GLT1 $(p<9.7 \mathrm{E}-03)$ (Fig. 1, Additional file 2: Figure S5). Most revealing is that M10 was upregulated specifically in ASO mice, and decreased to WT expression levels by NSC transplantation. The functional annotation of this module revealed significant association with mitochondria and apoptosis, and (GO:0055114, GO:0043066, JAM:002874), and perhaps most interestingly the Parkin-Ubiquitin Proteasomal System (hs_WP2359) which has been directly linked with the lysosomal degradation of mitochondria (mitophagy) [32, 60] (Additional files 7, 8, 9 and 14). The most connected gene of this module was Dnajc30 (Dna) (Hsp40) Homolog, Subfamily C, Member 30, alias WBSCR18), an intron-less gene encoding a member of the DNAJ/HSP40 molecular chaperones which has been implicated in mitochondrial DNA maintenance and replication and oxidative stress $[98,99]$. This gene has also been associated with Williams syndrome, a multisystem developmental disorder characterized by mild to moderate delays in cognitive development and intellectual disabilities [72]. An additional interesting hub gene in this module is Bloc1s1. Bloc1s1 is a component of the BLOC-1 complex, which is involved in the biogenesis of lysosome-related organelle, suggesting a potential influence on lysosomal degradation of aggregates or dysfunctional organelles, both of which have been strongly implicated in synucleinopathies $[17,34,50,106]$. In 


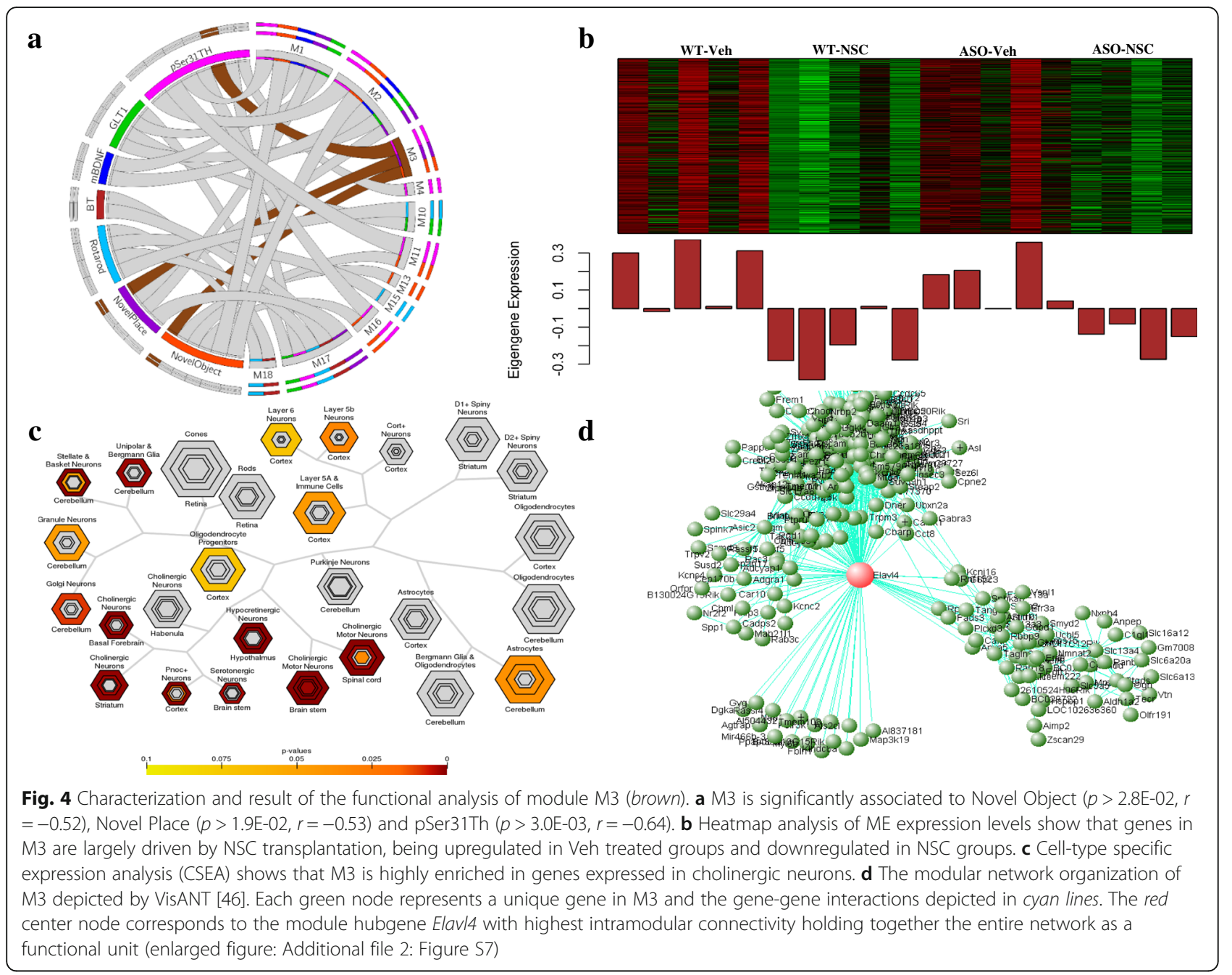

addition, the BLOC-1 complex is also involved in the negative regulation of aerobic respiration through mitochondrial protein lysine-acetylation [88, 89].

Another set of genes that further implicate mitochondrial and lysosomal function and autophagy are the 41 genes that make up Module 17 (M17, grey60) which clusters closely with M10 (Additional file 2: Figure S8) and shows a significant positive correlation with Novel Place recognition $(p<2.9 \mathrm{E}-04)$ and Rotarod $(p<4.3 \mathrm{E}-03)$, a negative correlation with Beam Traversal $(p<3.6 \mathrm{E}-03)$, and positive correlation with the biomarkers pSer31TH $(p<1.1 \mathrm{E}-02)$ and GLT1 $(p<2.3 \mathrm{E}-02)$ (Fig. 5a, Additional file 2: Figure S5). This module was upregulated in ASO mice compared to WT mice, and significantly downregulated in response to NSC transplantation in both WT and ASO mice (Fig. 5b) and is enriched in small nucleolar RNAs (GO:0005732), mitochondria-associated genes (GO:0005739, GO:0098798), histone acetyl transferase activity (GO:0035267), mTOR signaling and autophagy (mTORPathway) (Fig. 5c; Additional files 7, 8, 9 and 19).
Dysregulations of these processes have also been strongly implicated in synucleinopathies such as Parkinson's disease $[16,64]$.

\section{Discussion}

Due to the complex pathology of neurodegenerative disease, stem cells transplantation has been increasingly considered as a potential approach to compensate for neuronal loss, promote plasticity, and restore brain function $[15,24,57,63,69,96]$. While a growing number of studies support the potential translational value of stem cell therapies for neurodegeneration, the precise mechanisms by which these cells improve behavioral function remains largely unclear $[33,63,96]$. We previously reported that transplantation of murine NSCs leads to significant improvements in both motor and cognitive behavior in a transgenic model of Dementia with Lewy Bodies (DLB) that correlated with dopaminergic and glutamatergic biomarkers and were driven at least part by increases in mature BDNF protein [41]. However, viral 


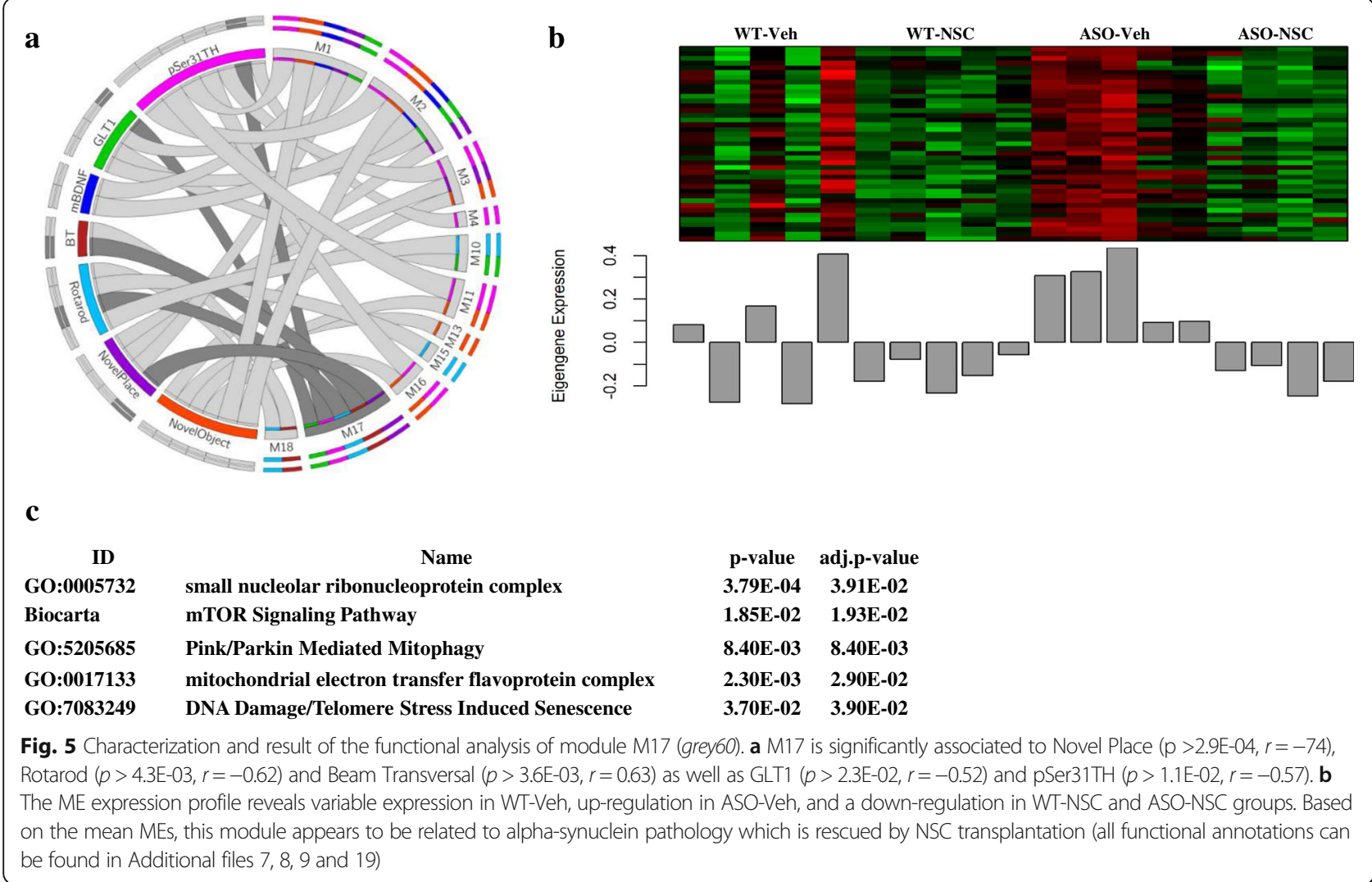

delivery of BDNF alone only partially mimicked the benefits of NSC transplantation, suggesting that other mechanisms are likely involved. To gain further insight into the multifactorial nature of stem cell therapy we therefore extended and complemented our previous experiment with gene expression network analysis to reveal transcriptional changes and cellular mechanisms that may underlie NSC related functional recovery in this model of DLB.

Correlational network analysis with comprehensive functional annotation provides a powerful approach to identify cell type specificity and related biochemical processes in complex systems such as NSCs transplanted into the CNS [65, 70, 104]. By comparing gene expression networks of striatal transcriptomes due to either DLB genotype or NSC transplantation, we detected several modules of highly connected genes that were significantly correlated with phenotypic changes. We report a diverse set of gene network modules that reflect changes ranging from large biological systemic changes (M1) to more focused and organelle specific changes (M17). This diversity of networks reflects the complex systemic changes that occur due to transplanting a biological system (NSCs) into another biological system (mouse CNS), and has allowed us to highlight participating clusters within larger altered networks [42, 70]. The functional annotation of genes in significant modules confirmed our previous observation that engrafted NSCs preferentially differentiate into astrocytes and oligodendrocytes. Likewise, we identified gene networks related to stem cell migration and proliferation consistent with our prior observation of robust NSC migration.

Interestingly, we also found evidence of gene enrichment associated with both pro- and anti-inflammatory state in response to NSC treatment. This antiinflammatory activation is most likely driven by the transplant itself as a previous report has demonstrated that newly formed glial cells in the CNS elevate antiinflammatory gene expression, counteracting proinflammatory cytokines and creating a more permissive environment for neuronal survival [54]. Therefore, it is possible that glial-differentiated NSCs or endogenous glial cells provide important anti-inflammatory signals following NSC transplantation. Although expression changes in genes associated with innate immunity were observed between ASO and WT mice, the $\alpha$-synuclein phenotype did not have a significant effect on NSCrelated gene networks. This finding, which is consistent with our prior report that NSC survival and differentiation is equivalent between ASO and WT mice, suggests that transplanted NSCs can differentiate normally regardless of $\alpha$-synuclein pathology (Additional files 4 and 5).

In addition to innate inflammatory responses, our results also point toward a role for the adaptive immune 
system in attenuating inflammation. Emerging evidence demonstrates that certain adaptive immune cells, such as $\mathrm{T}$ regulatory cells (Tregs) which have a pivotal role in maintaining immunological tolerance and inhibiting inflammatory responses, can play an important role in CNS repair $[4,7,23,51,54]$. For example, Chen et al. demonstrated that transplantation of human embryonic stem cell-derived neural precursor cells was associated with a reduction in neuroinflammation which correlated with an increased number of CD4(+)CD25(+)FOXP3(+) regulatory $\mathrm{T}$ cells and sustained clinical recovery in the mouse model of multiple sclerosis (MS) [23]. Moreover, our lab also recently described a potential cross-talk between $\mathrm{T}$ cells and microglia in an animal model of Alzheimer's disease further implicating the adaptive immune system in neurodegenerative disease [67]. It is therefore likely that in the ASO model, multiple cell populations contribute to either inflammatory or immunomodulatory conditions that influence behavior, and that these populations respond to and modulate one another via both cytokine expression and direct cell-to-cell interactions.

This unbiased network analysis of DLB mouse transcriptomes also provides an interesting perspective on $\alpha-$ synuclein related pathology, emphasizing aspects of pathology which can be rescued by NSC transplantation. Networks were enriched in genes associated with mitochondrial and lysosomal function, neurotransmission as well as synaptic plasticity. Mitochondrial dysfunction is prevalent in synucleinopathies as evidenced by clinical findings in patients with PD/DLB who carry genetic mutations of genes LRRK2 and PINK1 which are associated with mitochondrial function [38, 53, 100]. However, mitochondrial impairment can also result from $\alpha$ synuclein over-abundance independently of LRRK2 or PINK1 mutations [36, 68, 91]. Our functional annotation implied an over-active state of mitochondrial function in ASO mice characterized by an increase in oxidative stress, apoptosis and DNA damage that was somewhat diminished by NSC transplantation. Similar findings were described in transgenic mice overexpressing the A53T $\alpha$-synuclein mutation which showed regional associations between mutant protein and neuronal death, dysfunctional mitochondrial protein complex I, increased oxidative stress, and DNA damage [68]. Precisely, how NSC transplantation modulates these systems remains unclear, but these data nevertheless highlight an important new mechanism by which NSC transplantation may influence brain function.

One of the native functions of $\alpha$-synuclein is the modulation of vesicles at the pre-synaptic terminal, a process that is critical for synaptic signaling $[20,21,87$, 101]. Our network analysis identified several modules associated with changes in dopaminergic, cholinergic,
GABAergic, and glutamatergic neurotransmitter systems in response to NSC treatment. Those modules that were enriched in genes related to dopaminergic system also indicated an increased activity in D1 and D2 receptor expressing GABAergic medium spiny neurons as well as modulation in synaptic transmission, postsynaptic density and calcium signaling. These changes in neurotransmitter systems likely relate to our prior findings that mature BDNF produced by NSCs and astrocytes in the striatum is important for NSC-induced behavioral recovery [41]. Binding of BDNF to TrkB receptors which are expressed in striatal medium spiny neurons likely promotes the restoration of normal dendritic morphogenesis. Likewise, binding and subsequent retrograde transport of $\mathrm{BDNF} / \mathrm{TrkB}$ signaling endosomes in corticostriatal glutamatergic and nigrostriatal dopaminergic projections likely also influences the function and health of substance nigra and cortical neurons that project to the striatum $[10,113]$.

This analysis uncovered several interesting hub genes in connection to alpha-synuclein pathology and NSC related recovery including Itpr 1, which has been associated with dopaminergic and $\mathrm{Ca}^{2+}$ signaling. This gene encodes a ligand-gated ion channel, an intracellular receptor for inositol 1,4,5-trisphosphate molecules which is highly expressed in neurons [110] and deletions of Itpr1 are known to cause spinocerebellar ataxia [47]. Furthermore, activation of D1 dopamine receptors within the nucleus accumbens induces $\mathrm{Ca}^{2+}$ signals which are critical for neuronal excitability and synaptic plasticity [95]. Thus further investigation of Itpr1 functions in ASO mice could reveal additional insight about the potential dysregulation of $\mathrm{Ca}^{2+}$ homeostasis induced by alpha-synuclein pathology. Another interesting hub gene identified in our analysis is Elavl4 as several lines of evidence specify roles for this gene in neuronal plasticity, recovery from axonal injury, and learning and memory [84]. In addition, genetic variants in human ELAVL4 have been associated with age of onset in Parkinson disease (PD) [35]. Besides its role in mRNA stabilization in the brain, the functions of Elavl4 are still emerging. Therefore, future studies aimed at manipulating Elavl4 expression in the context of ASO mice and NSC transplantation could uncover important additional roles for this gene in DLB pathogenesis and NSCmediated behavioral recovery.

\section{Conclusion}

Taken together, our data suggest that NSC transplantation influences multiple gene networks and interacts with endogenous neural and immune cells to improve cognitive and motor behavior in DLB mice. Our analysis greatly extends our prior findings to implicate NSCinduced changes in synaptic plasticity, mitochondrial 
and lysosomal function, and both innate and adaptive immunity in functional recovery (Additional file 2: Figure S8). It also highlights the potential use of WGCNA analysis to uncover candidate genes such as Elavl1and Itpr1 that may be critically involved in the pathogenesis and/or potential treatment of DLB and warrant further investigation.

\section{Additional files}

Additional file 1: RNA_quality_measurments. Table S2. contains RNA related quality measurements including A260, A280, 260/280, 260/230 and RNA integrity number (RIN). (XLSX $11 \mathrm{~kb}$ )

Additional file 2: This file contains six supplemental figures. Figure S1. outlines neural stem cell transplantation strategy and WGCNA workflow. Figure S2. illustrates result of RNA degradation analysis. Figure S3. demonstrates the result of Quality Control (QC) Analysis of gene expression. Figure S4. illustrates a dendrogram produced by average linkage hierarchical clustering of approximately 12,00 genes based on the topological overlap matrix (TOM) as input similarities. Figure $\mathbf{S 5}$. visualizes the association between consensus module eigengenes and quantitative phenotypes related to NSC engraftment. Figure S6. depicts a heatmap, visualizing genotype specific gene expression pattern in association to innate immunity. Figure S7. shows an enlarged version of Fig. 4D. Figure S8. demonstrates a module eigengene (ME) network of 11 significant modules. (PDF $980 \mathrm{~kb}$ )

Additional file 3: Codes_and_Parameters. The documents contains codes and parameters used in WGCNA, GSEA and CSEA analysis. (PDF $56 \mathrm{~kb}$ )

Additional file 4: GSEA_result. The file contains the result of Gene Set Enrichment Analysis (GSEA) which detects coordinated changes in prespecified sets of related genes (13 modules) between treatment groups. Each tab of file indicates two conditions in which differential expression of each module as a gene set was tested (tab1: ASO-Veh vs ASO-NSC; tab2: ASO-NSC vs WT-Veh; tab3: WT-NSC vs WT-Veh; tab4 ASO-Veh vs WT-Veh; tab5 ASO-NSC vs WT-NSC). In each tab, first column indicates a module as gene set, the second column indicates the size of the module gene set and the following columns display GSEA statistics. Modules were considered statistically significant at FDR q-val < 0.05. (XLSX $137 \mathrm{~kb}$ )

Additional file 5: Pathways_altered_in_ASO_mice_and_NSC_ transplantation. Significant differentially regulated pathways between treatment groups summarized in Table S3. The analysis based on GSEA result and CluGo group term cluster annotation which determines the most representative $\mathrm{GO}$ term of a cluster of similar biological processes. The first column describes experimental group contrasts, the second columns indicates the significantly differentially expressed module gene sets (Additional file 4) between conditions; the third, fourth and fifth columns contain pathway information of annotated gene sets. Table contains potential dysregulated pathways associated with ASO genotype that are significantly different between ASO-Veh and WT-Veh conditions and pathways that are altered differently in ASO-Veh and ASO-NSC conditions. The overlap of pathway and Module eigegenes direction (Fig. 1, Additional file 4: tab6) between the two contrasts suggest that NSC treatment can alter ASO associated pathways. Pathways that are altered differently in ASO-NSC and WT-NSC conditions suggest an ASO genotype interaction with NSC treatment. (XLSX $359 \mathrm{~kb}$ )

Additional file 6: SignificantModules_geneKME_and_phenoCorrelation. Summary output of network analysis result. Each tab of the file is designated to a module. First column of the tab displays gene symbol of genes assigned to the module. Second and third colums indicate color assignment and module number. Fourth column contains intramodular connectivity (KME) (also known as degree) for each gene in the module and the rest of the columns correspond to correlation values of genes for each quantitative phenotypes. (XLSX $725 \mathrm{~kb}$ )

Additional file 7: ModuleAnnotation_internalCollection_enrichWGCNA. The file contains the most significant results, description and statistical information of enrichment analyses. Columns are labeled as followed: module number, module color, rank of the significant result, precomputed gene set IDs implemented in WGCNA (dataSetID), data set name (dataSetName), name of gene set library (inGroups), pValue, Bonferroni corrected $p$ value, FDR corrected $p$ value, the number pf overlapping genes between the gene set and the module (nCommonGenes), ratio of the overlapping genes and the size of module (fracOfEffectiveClassSize), module size (effectiveClassSize), ratio of overlapping genes and the gene set size (fracOfEffectiveSetSize), size of the gene set (effectiveSetSize), name of the gene set (shortDataSetName), symbols of the overlapping genes (overlapGenes). (XLSX $70 \mathrm{~kb}$ )

Additional file 8: ModuleAnnotation_CluGo. The result of enrichment analyses performed by CluGO in Cytoscape. ClueGO integrates Gene Ontology (GO) terms as well as pathways and creates a functionally organized GO-pathway term network [14]. Each tab of the file is designated to a module. The columns of each tabs correspond to the significant gene ontology (GO) terms with related single term and group (network) $p$-values in addition to genes associated with GO term. (XLSX 1913 kb)

Additional file 9: ModuleAnnotation_GOenrichmentWGCNA. Supplemantary Gene Ontology Annotation performed in WGCNA. This file contains the first 25 most significant annotation terms for each module based on Gene Ontology gene sets enrichment analysis implemented in WGCNA. Columns in the file are labeled as followed: rank of the significant result (rank), module number (module), module size (modSize), the number of gene from the module that are included in the analysis (bkgrModSize), the size of GO gene set (bkgrTermSize), the number of overlapping genes from the module with GO gene set (nModGenesInTerm), ratio of overlapping gene (nModGenesInTerm) and size of effective module (bkgrModSize), ratio of overlapping gene nModGenesInTerm) and size of GO gene set, enrichment $P$ value, Bonferroni corrected P, GO ID (termID), GO term, GO term name and GO term definition. (XLSX $81 \mathrm{~kb}$ )

Additional file 10: ModuleAnnotation_enrichR_M1. Annotation result of M1 performed in enrichR. EnrichR currently contains annotated gene sets from 102 gene set libraries organized in 8 categories. Details of the gene set libraries in EnrichrR can be found in publications [25, 56]. Each tab of the file contains gene sets of a particular library that was found to be enriched in the module. The first column represent the gene set (Term), the second column shows the ratio of overlapping module genes with the gene set (Overlap), the following four columns are related to statistics ( $P$-value, Adjusted $P$-value, Z-score, Combined Score) and the fifth column shows the symbols of the overlapping genes. The gene sets are ranked by combined score. (XLSX $2936 \mathrm{~kb}$ )

Additional file 11: ModuleAnnotation_enrichR_M2. Annotation result of M2 performed in enrichR. EnrichR currently contains annotated gene sets from 102 gene set libraries organized in 8 categories. Details of the gene set libraries in EnrichrR can be found in publications [25, 56]. Each tab of the file contains gene sets of a particular library that was found to be enriched in the module. The first column represent the gene set (Term), the second column shows the ratio of overlapping module genes with the gene set (Overlap), the following four columns are related to statistics ( $P$-value, Adjusted $P$-value, Z-score, Combined Score) and the fifth column shows the symbols of the overlapping genes. The gene sets are ranked by combined score. (XLSX $822 \mathrm{~kb}$ )

Additional file 12: ModuleAnnotation_enrichR_M3. Annotation result of M3 performed in enrichR. EnrichR currently contains annotated gene sets from 102 gene set libraries organized in 8 categories. Details of the gene set libraries in EnrichrR can be found in publications. Each tab of the file contains gene sets of a particular library that was found to be enriched in the module. The first column represent the gene set (Term), the second column shows the ratio of overlapping module genes with the gene set (Overlap), the following four columns are related to statistics ( $P$-value, Adjusted P-value, Z-score, Combined Score) and the fifth column shows the symbols of the overlapping genes. The gene sets are ranked by combined score. (XLSX $1032 \mathrm{~kb}$ )

Additional file 13: ModuleAnnotation_enrichR_M4. Annotation result of M4 performed in enrichR. EnrichR currently contains annotated gene sets from 102 gene set libraries organized in 8 categories. Details of the gene 
set libraries in EnrichrR can be found in publications [25, 56]. Each tab of the file contains gene sets of a particular library that was found to be enriched in the module. The first column represent the gene set (Term), the second column shows the ratio of overlapping module genes with the gene set (Overlap), the following four columns are related to statistics ( $P$-value, Adjusted $P$-value, Z-score, Combined Score) and the fifth column shows the symbols of the overlapping genes. The gene sets are ranked by combined score. (XLSX $671 \mathrm{~kb}$ )

Additional file 14: ModuleAnnotation_enrichR_M10. Annotation result of M10 performed in enrichR. EnrichR currently contains annotated gene sets from 102 gene set libraries organized in 8 categories. Details of the gene set libraries in EnrichrR can be found in publications [25, 56]. Each tab of the file contains gene sets of a particular library that was found to be enriched in the module. The first column represent the gene set (Term), the second column shows the ratio of overlapping module genes with the gene set (Overlap), the following four columns are related to statistics ( $P$-value, Adjusted $P$-value, Z-score, Combined Score) and the fifth column shows the symbols of the overlapping genes. The gene sets are ranked by combined score. (XLSX $350 \mathrm{~kb})$

Additional file 15: ModuleAnnotation_enrichR_M11. Annotation result of M11 performed in enrichR. Each tab of the file is the outcome of a gene set library enrichment analysis implemented in enrichR. (XLSX $433 \mathrm{~kb}$ )

Additional file 16: ModuleAnnotation_enrichR_M13. Annotation result of M13 performed in enrichR. EnrichR currently contains annotated gene sets from 102 gene set libraries organized in 8 categories. Details of the gene set libraries in EnrichrR can be found in publication [25, 56]. Each tab of the file contains gene sets of a particular library that was found to be enriched in the module. The first column represent the gene set (Term), the second column shows the ratio of overlapping module genes with the gene set (Overlap), the following four columns are related to statistics ( $P$-value, Adjusted $P$-value, Z-score, Combined Score) and the fifth column shows the symbols of the overlapping genes. The gene sets are ranked by combined score. (XLSX $216 \mathrm{~kb})$

Additional file 17: ModuleAnnotation_enrichR_M15. Annotation result of M15 performed in enrichR. EnrichR currently contains annotated gene sets from 102 gene set libraries organized in 8 categories. Details of the gene set libraries in EnrichrR can be found in publications [25,56]. Each tab of the file contains gene sets of a particular library that was found to be enriched in the module. The first column represent the gene set (Term), the second column shows the ratio of overlapping module genes with the gene set (Overlap), the following four columns are related to statistics ( $P$-value, Adjusted $P$-value, Z-score, Combined Score) and the fifth column shows the symbols of the overlapping genes. The gene sets are ranked by combined score. (XLSX $431 \mathrm{~kb}$ )

Additional file 18: ModuleAnnotation_enrichR_M16. Annotation result of M16 performed in enrichR. EnrichR currently contains annotated gene sets from 102 gene set libraries organized in 8 categories. Details of the gene set libraries in EnrichrR can be found in publications [25, 56]. Each tab of the file contains gene sets of a particular library that was found to be enriched in the module. The first column represent the gene set (Term), the second column shows the ratio of overlapping module genes with the gene set (Overlap), the following four columns are related to statistics ( $P$-value, Adjusted $P$-value, Z-score, Combined Score) and the fifth column shows the symbols of the overlapping genes. The gene sets are ranked by combined score. (XLSX $125 \mathrm{~kb}$ )

Additional file 19: ModuleAnnotation_enrichR_M17. Annotation result of M17 performed in enrichR. EnrichR currently contains annotated gene sets from 102 gene set libraries organized in 8 categories. Details of the gene set libraries in EnrichrR can be found in publications [25, 56]. Each tab of the file contains gene sets of a particular library that was found to be enriched in the module. The first column represent the gene set (Term), the second column shows the ratio of overlapping module genes with the gene set (Overlap), the following four columns are related to statistics ( $P$-value, Adjusted $P$-value, Z-score, Combined Score) and the fifth column shows the symbols of the overlapping genes. The gene sets are ranked by combined score. (XLSX $412 \mathrm{~kb})$

Additional file 20: ModuleAnnotation_enrichR_M18. Annotation result of M18 performed in enrichR. EnrichR currently contains annotated gene sets from 102 gene set libraries organized in 8 categories. Details of the gene set libraries in EnrichrR can be found in publications [25, 56]. Each tab of the file contains gene sets of a particular library that was found to be enriched in the module. The first column represent the gene set (Term), the second column shows the ratio of overlapping module genes with the gene set (Overlap), the following four columns are related to statistics (P-value, Adjusted P-value, Z-score, Combined Score) and the fifth column shows the symbols of the overlapping genes. The gene sets are ranked by combined score. (XLSX $72 \mathrm{~kb}$ )

\section{Abbreviations}

ASO: Overexpressed wild-type human a-synuclein; BDNF: Brain-derived neurotrophic factor; BT: Beam Transversal; DLB: Dementia with Lewy Body; GLT-1: Glutamate type I transporter; NO: Novel Object; NP: Novel Place; NSCs: Neural stem cells; WGCNA: Weighted co-expression network analysis

\section{Acknowledgments}

We gratefully acknowledge Dr. Eliezer Masliah for development of the original PDGF- $\beta$ line-D ASO model and providing colony founder mice. We also thank Jacqueline Caesar M.S. for assistance in RNA isolation and purification and the UCl Genomics High-Throughput Facility and especially Dr. Melanie Oakes for their support and helpful discussion. We also express our gratitude for UCI HPC assistance and especially Dr. Harry Mangalam and Garr Updegraff for their generous support, and the UCI Research Computing group especially Allen Schiano and Dana Roode. This work was supported by an NSF Fellowship (NRSG), NIH AG029378 and AG16573 (MBJ), and generous donors to the UCI Sue and Bill Gross Stem Cell Research Center.

\section{Availability of data and materials}

The data set supporting the results of this article is available in the GEO repository: GSE95546).

\section{Authors' contributions}

AL: Conception and design of study, execution of computational analysis, interpretation of the data, manuscript writing. NRSG: Conception and design of study, data collection, interpretation of the data, manuscript writing. MBJ: Conception and design of study, interpretation of the data, manuscript writing. All authors read and approved the final manuscript.

\section{Competing interests}

The authors declare that they have no competing interest.

\section{Author details}

${ }^{1}$ Sue and Bill Gross Stem Cell Research Center, University of California, Irvine, USA. ${ }^{2}$ Institute for Memory Impairments and Neurological Disorders, University of California, Irvine, USA. ${ }^{3}$ Department of Neurobiology \& Behavior, University of California, Irvine, USA.

Received: 6 January 2017 Accepted: 24 February 2017 Published online: 10 March 2017

\section{References}

1. Agell N, Bachs O, Rocamora N, Villalonga P (2002) Modulation of the Ras/ Raf/MEK/ERK pathway by $\mathrm{Ca}(2+)$, and calmodulin. Cell Signal 14(8):649-654

2. Ager RR, Davis JL, Agazaryan A, Benavente F, Poon WW, LaFerla FM, BlurtonJones M (2015) Human neural stem cells improve cognition and promote synaptic growth in two complementary transgenic models of Alzheimer's disease and neuronal loss. Hippocampus 25(7):813-826

3. Albert R (2007) Network inference, analysis, and modeling in systems biology. Plant Cell 19(11):3327-3338

4. Amor S, Woodroofe MN (2014) Innate and adaptive immune responses in neurodegeneration and repair. Immunology 141(3):287-291

5. Balderas I, Rodriguez-Ortiz CJ, Salgado-Tonda P, Chavez-Hurtado J, McGaugh JL, Bermudez-Rattoni F (2008) The consolidation of object and context recognition memory involve different regions of the temporal lobe. Learn Mem 15(9):618-624

6. Barker GR, Bird F, Alexander V, Warburton EC (2007) Recognition memory for objects, place, and temporal order: a disconnection analysis of the role of the medial prefrontal cortex and perirhinal cortex. J Neurosci 27(11):2948-2957 
7. Baruch K, Rosenzweig N, Kertser A, Deczkowska A, Sharif AM, Spinrad A, Tsitsou-Kampeli A, Sarel A, Cahalon L, Schwartz M (2015) Breaking immune tolerance by targeting Foxp3(+) regulatory T cells mitigates Alzheimer's disease pathology. Nat Commun 6:7967

8. Bastian M, Heymann S, J M (2009) Gephi: an open source software for exploring and manipulating networks. AAAI Publications.

9. Batiz LF, Castro MA, Burgos PV, Velasquez ZD, Munoz RI, Lafourcade CA, Troncoso-Escudero P, Wyneken U (2015) Exosomes as novel regulators of adult neurogenic niches. Front Cell Neurosci 9:501

10. Baydyuk M, Xu B (2014) BDNF signaling and survival of striatal neurons. Front Cell Neurosci 8:254

11. Bermudez-Rattoni F, Okuda S, Roozendaal B, McGaugh JL (2005) Insular cortex is involved in consolidation of object recognition memory. Learn Mem 12(5):447-449

12. Berridge MJ (1993) Inositol trisphosphate and calcium signalling. Nature 361(6410):315-325

13. Beyer K, Domingo-Sabat M, Ariza A (2009) Molecular pathology of Lewy body diseases. Int J Mol Sci 10(3):724-745

14. Bindea G, Mlecnik B, Hackl H, Charoentong P, Tosolini M, Kirilovsky A, Fridman WH, Pages F, Trajanoski Z, Galon J (2009) ClueGO: a Cytoscape plug-in to decipher functionally grouped gene ontology and pathway annotation networks. Bioinformatics 25(8):1091-1093

15. Blurton-Jones M, Kitazawa M, Martinez-Coria H, Castello NA, Muller FJ, Loring JF, Yamasaki TR, Poon WW, Green KN, LaFerla FM (2009) Neural stem cells improve cognition via BDNF in a transgenic model of Alzheimer disease. Proc Natl Acad Sci U S A 106(32):13594-13599

16. Bockaert J, Marin P (2015) mTOR in brain physiology and pathologies. Physiol Rev 95(4):1157-1187

17. Bourdenx M, Bezard E, Dehay B (2014) Lysosomes and alpha-synuclein form a dangerous duet leading to neuronal cell death. Front Neuroanat 8:83

18. Breuer K, Foroushani AK, Laird MR, Chen C, Sribnaia A, Lo R, Winsor GL, Hancock RE, Brinkman FS, Lynn DJ (2013) InnateDB: systems biology of innate immunity and beyond-recent updates and continuing curation. Nucleic Acids Res 41(Database issue):D1228-D1233

19. Bronicki LM, Jasmin BJ (2013) Emerging complexity of the HuD/ELAVI4 gene; implications for neuronal development, function, and dysfunction. RNA 19(8):1019-1037

20. Burre J (2015) The synaptic function of alpha-synuclein. J Parkinsons Dis 5(4):699-713

21. Burre J, Sharma M, Tsetsenis T, Buchman V, Etherton MR, Sudhof TC (2010) Alpha-synuclein promotes SNARE-complex assembly in vivo and in vitro. Science 329(5999):1663-1667

22. Castro MA, Wang X, Fletcher MN, Meyer KB, Markowetz F (2012) RedeR: R/ Bioconductor package for representing modular structures, nested networks and multiple levels of hierarchical associations. Genome Biol 13(4):R29

23. Chen L, Coleman R, Leang R, Tran H, Kopf A, Walsh CM, Sears-Kraxberger I, Steward O, Macklin WB, Loring JF, Lane TE (2014) Human neural precursor cells promote neurologic recovery in a viral model of multiple sclerosis. Stem Cell Reports 2(6):825-837

24. Chen LW, Kuang F, Wei LC, Ding YX, Yung KK, Chan YS (2011) Potential application of induced pluripotent stem cells in cell replacement therapy for Parkinson's disease. CNS Neurol Disord Drug Targets 10(4):449-458

25. Chen EY, Tan CM, Kou Y, Duan Q, Wang Z, Meirelles GV, Clark NR, Ma'ayan A (2013) Enrichr: interactive and collaborative HTML5 gene list enrichment analysis tool. BMC Bioinformatics 14:128

26. Chuderland D, Seger R (2008) Calcium regulates ERK signaling by modulating its protein-protein interactions. Commun Integr Biol 1(1):4-5

27. Cossetti C, Iraci N, Mercer TR, Leonardi T, Alpi E, Drago D, Alfaro-Cervello C, Saini HK, Davis MP, Schaeffer J, Vega B, Stefanini M, Zhao C, Muller W, Garcia-Verdugo JM, Mathivanan S, Bachi A, Enright AJ, Mattick JS, Pluchino S (2014) Extracellular vesicles from neural stem cells transfer IFN-gamma via Ifngr1 to activate Stat1 signaling in target cells. Mol Cell 56(2):193-204

28. Csárdi G, Nepusz T (2006) The igraph software package for complex network research. Inter Journal Complex Systems.

29. Danik JS, Pare G, Chasman DI, Zee RY, Kwiatkowski DJ, Parker A, Miletich JP, Ridker PM (2009) Novel loci, including those related to Crohn disease, psoriasis, and inflammation, identified in a genome-wide association study of fibrinogen in 17686 women: the Women's Genome Health Study. Circ Cardiovasc Genet 2(2):134-141

30. De Strooper B (2014) Lessons from a failed gamma-secretase Alzheimer trial. Cell 159(4):721-726
31. De Toro J, Herschlik L, Waldner C, Mongini C (2015) Emerging roles of exosomes in normal and pathological conditions: new insights for diagnosis and therapeutic applications. Front Immunol 6:203

32. Deas E, Wood NW, Plun-Favreau H (2011) Mitophagy and Parkinson's disease: the PINK1-parkin link. Biochim Biophys Acta 1813(4):623-633

33. Decimo I, Bifari F, Krampera M, Fumagalli G (2012) Neural stem cell niches in health and diseases. Curr Pharm Des 18(13):1755-1783

34. Dehay B, Martinez-Vicente M, Caldwell GA, Caldwell KA, Yue Z, Cookson MR, Klein C, Vila M, Bezard E (2013) Lysosomal impairment in Parkinson's disease. Mov Disord 28(6):725-732

35. DeStefano AL, Latourelle J, Lew MF, Suchowersky O, Klein C, Golbe LI, Mark MH, Growdon JH, Wooten GF, Watts R, Guttman M, Racette BA, Perlmutter JS, Marlor L, Shill HA, Singer C, Goldwurm S, Pezzoli G, SaintHilaire MH, Hendricks AE, Gower A, Williamson S, Nagle MW, Wilk JB, Massood T, Huskey KW, Baker KB, Itin I, Litvan I, Nicholson G, Corbett A Nance M, Drasby E, Isaacson S, Burn DJ, Chinnery PF, Pramstaller PP, AlHinti J, Moller AT, Ostergaard K, Sherman SJ, Roxburgh R, Snow B, Slevin JT, Cambi F, Gusella JF, Myers RH (2008) Replication of association between ELAVL4 and Parkinson disease: the GenePD study. Hum Genet 124(1):95-99

36. Devi L, Raghavendran V, Prabhu BM, Avadhani NG, Anandatheerthavarada HK (2008) Mitochondrial import and accumulation of alpha-synuclein impair complex I in human dopaminergic neuronal cultures and Parkinson disease brain. J Biol Chem 283(14):9089-9100

37. Garcia-Reitbock P, Anichtchik O, Bellucci A, lovino M, Ballini C, Fineberg E, Ghetti B, Della Corte L, Spano P, Tofaris GK, Goedert M, Spillantini MG (2010) SNARE protein redistribution and synaptic failure in a transgenic mouse model of Parkinson's disease. Brain 133(Pt 7):2032-2044

38. Gilks WP, Abou-Sleiman PM, Gandhi S, Jain S, Singleton A, Lees AJ, Shaw K, Bhatia KP, Bonifati V, Quinn NP, Lynch J, Healy DG, Holton JL, Revesz T, Wood NW (2005) A common LRRK2 mutation in idiopathic Parkinson's disease. Lancet 365(9457):415-416

39. Giusto E, Donega M, Cossetti C, Pluchino S (2014) Neuro-immune interactions of neural stem cell transplants: from animal disease models to human trials. Exp Neurol 260:19-32

40. Goldberg NRS, Blurton-Jones M (2015) Can stem cells be used to enhance cognition? In: Knafo S, Venero C (Eds.), Cognitive Enhancement: Pharmacologic, Environmental and Genetic Factors, pp 167-192

41. Goldberg NR, Caesar J, Park A, Sedgh S, Finogenov G, Masliah E, Davis J, Blurton-Jones M (2015) Neural stem cells rescue cognitive and motor dysfunction in a transgenic model of dementia with lewy bodies through a BDNF-dependent mechanism. Stem Cell Reports 5(5):791-804

42. Gupta S, Ellis SE, Ashar FN, Moes A, Bader JS, Zhan J, West AB, Arking DE (2014) Transcriptome analysis reveals dysregulation of innate immune response genes and neuronal activity-dependent genes in autism. Nat Commun 5:5748

43. Halbleib JM, Nelson WJ (2006) Cadherins in development: cell adhesion, sorting, and tissue morphogenesis. Genes Dev 20(23):3199-3214

44. Helms C, Cao L, Krueger JG, Wijsman EM, Chamian F, Gordon D, Heffernan M, Daw JA, Robarge J, Ott J, Kwok PY, Menter A, Bowcock AM (2003) A putative RUNX1 binding site variant between SLC9A3R1 and NAT9 is associated with susceptibility to psoriasis. Nat Genet 35(4):349-356

45. Holtman IR, Raj DD, Miller JA, Schaafsma W, Yin Z, Brouwer N, Wes PD, Moller T, Orre M, Kamphuis W, Hol EM, Boddeke EW, Eggen BJ (2015) Induction of a common microglia gene expression signature by aging and neurodegenerative conditions: a co-expression meta-analysis. Acta Neuropathol Commun 3:31

46. Hu Z, Chang YC, Wang Y, Huang CL, Liu Y, Tian F, Granger B, Delisi C (2013) VisANT 4.0: integrative network platform to connect genes, drugs, diseases and therapies. Nucleic Acids Res 41(Web Server issue):W225-W231

47. Huang L, Chardon JW, Carter MT, Friend KL, Dudding TE, Schwartzentruber J, Zou R, Schofield PW, Douglas S, Bulman DE, Boycott KM (2012) Missense mutations in ITPR1 cause autosomal dominant congenital nonprogressive spinocerebellar ataxia. Orphanet J Rare Dis 7:67

48. Hulpiau P, van Roy F (2009) Molecular evolution of the cadherin superfamily. Int J Biochem Cell Biol 41(2):349-369

49. Ito K, Murphy D (2013) Application of ggplot2 to pharmacometric graphics. CPT Pharmacometrics Syst Pharmacol 2:e79

50. John Peter AT, Lachmann J, Rana M, Bunge M, Cabrera M, Ungermann C (2013) The BLOC-1 complex promotes endosomal maturation by recruiting the Rab5 GTPase-activating protein Msb3. J Cell Biol 201(1):97-111 
51. Josefowicz SZ, Lu LF, Rudensky AY (2012) Regulatory T cells: mechanisms of differentiation and function. Annu Rev Immunol 30:531-564

52. Kalra $H$, Simpson RJ, Ji H, Aikawa E, Altevogt $P$, Askenase $P$, Bond VC, Borras FE, Breakefield X, Budnik V, Buzas E, Camussi G, Clayton A, Cocucci E, Falcon-Perez JM, Gabrielsson S, Gho YS, Gupta D, Harsha HC, Hendrix A, Hill AF, Inal JM, Jenster G, Kramer-Albers EM, Lim SK, Llorente A, Lotvall J, Marcilla A, Mincheva-Nilsson L, Nazarenko I, Nieuwland R, Nolte-'t Hoen EN, Pandey A, Patel T, Piper MG, Pluchino S, Prasad TS, Rajendran L, Raposo G, Record M, Reid GE, Sanchez-Madrid F, Schiffelers RM, Siljander P, Stensballe A, Stoorvogel W, Taylor D, Thery C, Valadi H, van Balkom BW, Vazquez J, Vidal M, Wauben MH, Yanez-Mo M, Zoeller M, Mathivanan S (2012) Vesiclepedia: a compendium for extracellular vesicles with continuous community annotation. PLoS Biol 10(12):e1001450

53. Klein C, Westenberger A (2012) Genetics of Parkinson's disease. Cold Spring Harb Perspect Med 2(1):a008888

54. Kokaia Z, Martino G, Schwartz M, Lindvall O (2012) Cross-talk between neural stem cells and immune cells: the key to better brain repair? Nat Neurosci 15(8):1078-1087

55. Krzywinski M, Schein J, Birol I, Connors J, Gascoyne R, Horsman D, Jones SJ, Marra MA (2009) Circos: an information aesthetic for comparative genomics. Genome Res 19(9):1639-1645

56. Kuleshov MV, Jones MR, Rouillard AD, Fernandez NF, Duan Q, Wang Z, Koplev S, Jenkins SL, Jagodnik KM, Lachmann A, McDermott MG, Monteiro CD, Gundersen GW, Ma'ayan A (2016) Enrichr: a comprehensive gene set enrichment analysis web server 2016 update. Nucleic Acids Res 44(W1): W90-W97

57. Lane EL, Handley OJ, Rosser AE, Dunnett SB (2008) Potential cellular and regenerative approaches for the treatment of Parkinson's disease. Neuropsychiatr Dis Treat 4(5):835-845

58. Langfelder P, Horvath S (2008) WGCNA: an R package for weighted correlation network analysis. BMC Bioinformatics 9:559

59. Langfelder P, Mischel PS, Horvath S (2013) When is hub gene selection better than standard meta-analysis? PLoS One 8(4):e61505

60. Lazarou M, Sliter DA, Kane LA, Sarraf SA, Wang C, Burman JL, Sideris DP, Fogel Al, Youle RJ (2015) The ubiquitin kinase PINK1 recruits autophagy receptors to induce mitophagy. Nature 524(7565):309-314

61. Le Blon D, Guglielmetti C, Hoornaert C, Quarta A, Daans J, Dooley D, Lemmens E, Praet J, De Vocht N, Reekmans K, Santermans E, Hens N, Goossens H, Verhoye M, Van der Linden A, Berneman Z, Hendrix S, Ponsaerts P (2016) Intracerebral transplantation of interleukin 13-producing mesenchymal stem cells limits microgliosis, oligodendrocyte loss and demyelination in the cuprizone mouse model. J Neuroinflammation 13(1):288

62. Lee SY, Min HK, Lee SH, Shin HJ, Lee WY, Cho YG, Kwok SK, Ju JH, Cho ML, Park SH (2016) IL-1 receptor antagonist (IL-1Ra)-Fc ameliorate autoimmune arthritis by regulation of the Th17 cells/Treg balance and arthrogenic cytokine activation. Immunol Lett 172:56-66

63. Levy M, Boulis N, Rao M, Svendsen CN (2016) Regenerative cellular therapies for neurologic diseases. Brain Res 1638(Pt A):88-96

64. Li J, Li S, Zhang L, Ouyang L, Liu B (2015) Deconvoluting the complexity of autophagy and Parkinson's disease for potential therapeutic purpose. Oncotarget 6(38):40480-40495

65. Li H, Xuan J, Wang Y, Zhan M (2008) Inferring regulatory networks. Front Biosci 13:263-275

66. Lindvall O, Barker RA, Brustle O, Isacson O, Svendsen CN (2012) Clinical translation of stem cells in neurodegenerative disorders. Cell Stem Cell 10(2):151-155

67. Marsh SE, Abud EM, Lakatos A, Karimzadeh A, Yeung ST, Davtyan H, Fote GM, Lau L, Weinger JG, Lane TE, Inlay MA, Poon WW, Blurton-Jones M (2016) The adaptive immune system restrains Alzheimer's disease pathogenesis by modulating microglial function. Proc Natl Acad Sci U S A 113(9):E1316-E1325

68. Martin LJ, Pan Y, Price AC, Sterling W, Copeland NG, Jenkins NA, Price DL, Lee MK (2006) Parkinson's disease alpha-synuclein transgenic mice develop neuronal mitochondrial degeneration and cell death. J Neurosci 26(1):41-50

69. Martino G, Pluchino S (2006) The therapeutic potential of neural stem cells. Nat Rev Neurosci 7(5):395-406

70. Mason MJ, Fan G, Plath K, Zhou Q, Horvath S (2009) Signed weighted gene co-expression network analysis of transcriptional regulation in murine embryonic stem cells. BMC Genomics 10:327
71. McNulty SE, Barrett RM, Vogel-Ciernia A, Malvaez M, Hernandez N, Davatolhagh MF, Matheos DP, Schiffman A, Wood MA (2012) Differential roles for Nr4a1 and Nr4a2 in object location vs. object recognition longterm memory. Learn Mem 19(12):588-592

72. Merla G, Ucla C, Guipponi M, Reymond A (2002) Identification of additional transcripts in the Williams-Beuren syndrome critical region. Hum Genet 110(5):429-438

73. Miller JA, Oldham MC, Geschwind DH (2008) A systems level analysis of transcriptional changes in Alzheimer's disease and normal aging. J Neurosci 28(6):1410-1420

74. Miller JA, Woltjer RL, Goodenbour JM, Horvath S, Geschwind DH (2013) Genes and pathways underlying regional and cell type changes in Alzheimer's disease. Genome Med 5(5):48

75. Mizumoto H, Mizumoto K, Shatos MA, Klassen H, Young MJ (2003) Retinal transplantation of neural progenitor cells derived from the brain of GFP transgenic mice. Vision Res 43(16):1699-1708

76. Network and Pathway Analysis Subgroup of Psychiatric Genomics Consortium (2015) Psychiatric genome-wide association study analyses implicate neuronal, immune and histone pathways. Nat Neurosci 18(2):199-209

77. Oldham MC, Langfelder P, Horvath S (2012) Network methods for describing sample relationships in genomic datasets: application to Huntington's disease. BMC Syst Biol 6:63

78. Ouyang W, Beckett O, Ma Q, Paik JH, DePinho RA, Li MO (2010) Foxo proteins cooperatively control the differentiation of Foxp3+ regulatory $\mathrm{T}$ cells. Nat Immunol 11(7):618-627

79. Palma JA, Kaufmann H (2014) Autonomic disorders predicting Parkinson's disease. Parkinsonism Relat Disord 20(Suppl 1):S94-S98

80. Parikshak NN, Gandal MJ, Geschwind DH (2015) Systems biology and gene networks in neurodevelopmental and neurodegenerative disorders. Nat Rev Genet 16(8):441-458

81. Pathan M, Keerthikumar S, Ang CS, Gangoda L, Quek CY, Williamson NA, Mouradov D, Sieber OM, Simpson RJ, Salim A, Bacic A, Hill AF, Stroud DA, Ryan MT, Agbinya Jl, Mariadason JM, Burgess AW, Mathivanan S (2015) FunRich: an open access standalone functional enrichment and interaction network analysis tool. Proteomics 15(15):2597-2601

82. Pluchino S, Cossetti C (2013) How stem cells speak with host immune cells in inflammatory brain diseases. Glia 61(9):1379-1401

83. Potkin SG, Turner JA, Guffanti G, Lakatos A, Torri F, Keator DB, Macciardi F (2009) Genome-wide strategies for discovering genetic influences on cognition and cognitive disorders: methodological considerations. Cogn Neuropsychiatry 14(4-5):391-418

84. Routtenberg A, Cantallops I, Zaffuto S, Serrano P, Namgung U (2000) Enhanced learning after genetic overexpression of a brain growth protein Proc Natl Acad Sci U S A 97(13):7657-7662

85. Sakaguchi S, Ono M, Setoguchi R, Yagi H, Hori S, Fehervari Z, Shimizu J, Takahashi T, Nomura T (2006) Foxp3+ CD25+ CD4+ natural regulatory T cells in dominant self-tolerance and autoimmune disease. Immunol Rev 212:8-27

86. Salvatore MF (2014) ser31 Tyrosine hydroxylase phosphorylation parallels differences in dopamine recovery in nigrostriatal pathway following 6OHDA lesion. J Neurochem 129(3):548-558

87. Schulz-Schaeffer WJ (2010) The synaptic pathology of alpha-synuclein aggregation in dementia with Lewy bodies, Parkinson's disease and Parkinson's disease dementia. Acta Neuropathol 120(2):131-143

88. Scott I, Webster BR, Li JH, Sack MN (2012) Identification of a molecular component of the mitochondrial acetyltransferase programme: a novel role for GCN5L1. Biochem J 443(3):655-661

89. Setty SR, Tenza D, Truschel ST, Chou E, Sviderskaya EV, Theos AC, Lamoreux ML, Di Pietro SM, Starcevic M, Bennett DC, Dell'Angelica EC, Raposo G, Marks MS (2007) BLOC-1 is required for cargo-specific sorting from vacuolar early endosomes toward lysosome-related organelles. Mol Biol Cell 18(3):768-780

90. Sevigny J, Chiao P, Bussiere T, Weinreb PH, Williams L, Maier M, Dunstan R, Salloway S, Chen T, Ling Y, O'Gorman J, Qian F, Arastu M, Li M, Chollate S, Brennan MS, Quintero-Monzon O, Scannevin RH, Arnold HM, Engber T, Rhodes K, Ferrero J, Hang Y, Mikulskis A, Grimm J, Hock C, Nitsch RM, Sandrock A (2016) The antibody aducanumab reduces Abeta plaques in Alzheimer's disease. Nature 537(7618):50-56

91. Sherer TB, Betarbet R, Stout AK, Lund S, Baptista M, Panov AV, Cookson MR, Greenamyre JT (2002) An in vitro model of Parkinson's disease: linking mitochondrial impairment to altered alpha-synuclein metabolism and oxidative damage. J Neurosci 22(16):7006-7015 
92. Shoemaker LD, Kornblum HI (2016) Neural stem cells (NSCs) and proteomics. Mol Cell Proteomics 15(2):344-354

93. Subramanian A, Kuehn H, Gould J, Tamayo P, Mesirov JP (2007) GSEA-P: a desktop application for Gene Set Enrichment Analysis. Bioinformatics 23(23):3251-3253

94. Subramanian A, Tamayo P, Mootha VK, Mukherjee S, Ebert BL, Gillette MA Paulovich A, Pomeroy SL, Golub TR, Lander ES, Mesirov JP (2005) Gene set enrichment analysis: a knowledge-based approach for interpreting genomewide expression profiles. Proc Natl Acad Sci U S A 102(43):15545-15550

95. Swapna I, Bondy B, Morikawa H (2016) Differential dopamine regulation of $\mathrm{Ca}(2+)$ signaling and its timing dependence in the nucleus accumbens. Cell Rep 15(3):563-573

96. Thompson LH, Bjorklund A (2015) Reconstruction of brain circuitry by neural transplants generated from pluripotent stem cells. Neurobiol Dis 79:28-40

97. Tofaris GK (2012) Lysosome-dependent pathways as a unifying theme in Parkinson's disease. Mov Disord 27(11):1364-1369

98. Tyc J, Klingbeil MM, Lukes J (2015) Mitochondrial heat shock protein machinery hsp70/hsp40 is indispensable for proper mitochondrial DNA maintenance and replication. mBio 6(1):e02425-14.

99. Uhrigshardt $H$, Singh A, Kovtunovych G, Ghosh M, Rouault TA (2010) Characterization of the human HSC20, an unusual DnaJ type III protein, involved in iron-sulfur cluster biogenesis. Hum Mol Genet 19(19):3816-3834

100. Valente EM, Abou-Sleiman PM, Caputo V, Muqit MM, Harvey K, Gispert S, Ali Z, Del Turco D, Bentivoglio AR, Healy DG, Albanese A, Nussbaum R, Gonzalez-Maldonado R, Deller T, Salvi S, Cortelli P, Gilks WP, Latchman DS, Harvey RJ, Dallapiccola B, Auburger G, Wood NW (2004) Hereditary earlyonset Parkinson's disease caused by mutations in PINK1. Science 304(5674): 1158-1160

101. Vargas KJ, Makani S, Davis T, Westphal CH, Castillo PE, Chandra SS (2014) Synucleins regulate the kinetics of synaptic vesicle endocytosis. J Neurosci 34(28):9364-9376

102. Visanji N, Marras C (2015) The relevance of pre-motor symptoms in Parkinson's disease. Expert Rev Neurother 15(10):1205-1217

103. Wes PD, Holtman IR, Boddeke EW, Moller T, Eggen BJ (2016) Next generation transcriptomics and genomics elucidate biological complexity of microglia in health and disease. Glia 64(2):197-213

104. Winden KD, Oldham MC, Mirnics K, Ebert PJ, Swan CH, Levitt P, Rubenstein $J$, Horvath S, Geschwind DH (2009) The organization of the transcriptional network in specific neuronal classes. Mol Syst Biol 5:291

105. Wiwatwattana N, Landau CM, Cope GJ, Harp GA, Kumar A (2007) Organelle DB: an updated resource of eukaryotic protein localization and function. Nucleic Acids Res 35(Database issue):D810-D814

106. Xilouri M, Brekk OR, Stefanis L (2016) Autophagy and alpha-synuclein: relevance to Parkinson's disease and related synucleopathies. Mov Disord 31(2):178-192

107. Xilouri M, Stefanis L (2011) Autophagic pathways in Parkinson disease and related disorders. Expert Rev Mol Med 13:e8

108. Xu X, Wells AB, O'Brien DR, Nehorai A, Dougherty JD (2014) Cell typespecific expression analysis to identify putative cellular mechanisms for neurogenetic disorders. J Neurosci 34(4):1420-1431

109. Yu G, Smith DK, Zhu H, Guan Y, Lam TT (2016) ggtree: an R package for visualization and annotation of phylogenetic trees with their covariates and other associated data. Methods Ecol Evol 8(1):28-36. http://dx.doi.org/ 10.1111/2041-210x.12628

110. Zhang Y, Chen K, Sloan SA, Bennett ML, Scholze AR, O'Keeffe S, Phatnani HP, Guarnieri P, Caneda C, Ruderisch N, Deng S, Liddelow SA, Zhang C, Daneman R, Maniatis T, Barres BA, Wu JQ (2014) An RNA-sequencing transcriptome and splicing database of glia, neurons, and vascular cells of the cerebral cortex. J Neurosci 34(36):11929-11947

111. Zhang H, Duan C, Yang H (2015) Defective autophagy in Parkinson's disease: lessons from genetics. Mol Neurobiol 51(1):89-104

112. Zhang B, Gaiteri C, Bodea LG, Wang Z, McElwee J, Podtelezhnikov AA, Zhang C, Xie T, Tran L, Dobrin R, Fluder E, Clurman B, Melquist S, Narayanan M, Suver C, Shah H, Mahajan M, Gillis T, Mysore J, MacDonald ME, Lamb JR, Bennett DA, Molony C, Stone DJ, Gudnason V, Myers AJ, Schadt EE, Neumann H, Zhu J, Emilsson V (2013) Integrated systems approach identifies genetic nodes and networks in late-onset Alzheimer's disease. Cell 153(3):707-720
113. Zhou B, Cai Q, Xie Y, Sheng ZH (2012) Snapin recruits dynein to BDNF-TrkB signaling endosomes for retrograde axonal transport and is essential for dendrite growth of cortical neurons. Cell Rep 2(1):42-51

114. Zou X, Ci HL, Chen W, Li YP (2006) [Cloning and expression analysis of human $\mathrm{N}$-acetyltransferase doman containing gene hNATL]. Fen Zi Xi Bao Sheng Wu Xue Bao 39(1):22-28

\section{Submit your next manuscript to BioMed Central and we will help you at every step:}

- We accept pre-submission inquiries

- Our selector tool helps you to find the most relevant journal

- We provide round the clock customer support

- Convenient online submission

- Thorough peer review

- Inclusion in PubMed and all major indexing services

- Maximum visibility for your research

Submit your manuscript at www.biomedcentral.com/submit

) Biomed Central 